\title{
On the Ramsey Equilibrium with Heterogeneous Consumers and Endogenous Labor Supply*
}

\author{
Stefano $\mathrm{BOSI}^{\dagger}$ and Thomas SEEGMULLER ${ }^{\ddagger}$
}

January 8, 2007

\begin{abstract}
In this paper we address the question of deterministic cycles in a Ramsey model with heterogeneous infinite-lived agents and borrowing constraints, augmented to take into account the case of elastic labor supply. Under usual restrictions, not only we show that the steady state is unique, but also we clarify its stability properties through a local analysis. We find that, in many cases, the introduction of elastic labor supply promotes convergence by widening the range of parameters for saddle-path stability, and endogenous cycles can eventually disappear. These results are robustly illustrated by means of canonical examples in which consumers have separable, KPR or homogeneous preferences.
\end{abstract}

JEL classification: C62, D30, E32.

Keywords: Saddle-path stability, endogenous cycles, heterogeneous agents, endogenous labor supply, borrowing constraint.

\footnotetext{
*This work was supported by French National Research Agency Grant (ANR-05-BLAN0347-01).

${ }^{\dagger}$ EQUIPPE, Département d'Economie, Université de Lille 1, Bât. SH2, Cité Scientifique, 59655 Villeneuve d'Ascq Cedex, France. Tel: (33) 3203370 12. EPEE, Université d'Evry. E-mail: stefano.bosi@univ-lille1.fr.

${ }^{\ddagger}$ CNRS and Centre d'Economie de la Sorbonne, Paris School of Economics, Université Paris 1, 106-112, bd. de l'Hôpital, 75647 Paris cedex 13, France. Tel: (33) 1440781 99. Fax: (33) 1440782 31. E-mail: seegmu@univ-paris1.fr.
} 


\section{Introduction}

Growth theorists are usually confronted with the question of convergence of an economic system. In neoclassical model of capital accumulation, commonly addressed questions are whether economies converge to the same long-run equilibrium, how convergence takes place and whether is monotonic, how fundamentals affect the stability properties of equilibrium. ${ }^{1}$

The most influential growth model is undoubtedly Ramsey which is characterized, in its basic version, by a representative agent with exogenous labor supply, saddle-path stability, equilibrium uniqueness and optimality. In order to add a degree of realism, economic literature has not only introduced various kinds of market imperfections, but also considered elastic labor supply and agents' heterogeneity.

On the one side, a class of papers have been devoted in the last decade to shed a light on the interplay between the consumption-labor arbitrage and the mechanism of capital accumulation in models with a representative consumer. The reader is referred, among others, to De Hek (1998), a one-sector model with an infinite-lived agent; to Bosi, Magris and Venditti (2005), a two-sector model; to Ladron-de-Guevara, Ortiguiera and Santos (1997), an endogenous growth model; to Nourry (2001) and Nourry and Venditti (2006), overlapping generations economies; to, eventually, Pintus (2006) and Garnier, Nishimura and Venditti (2006), models with externalities.

On the other side, there are papers with heterogeneous consumers which focus on the convergence of capital accumulation and distribution in the long run. Unlike the case with a representative agent, consumers' heterogeneity promotes borrowing transactions. The existence of borrowing constraints plays a key role on the dynamics of capital accumulation. Becker (1980), Becker and Foias $(1987,1994)$ and Sorger (1994) study economies where agents discount future differently, while Hernandez (1991) consider heterogenous but equally patient consumers.

Surprisingly, few works have investigated the role of elastic labor supply on equilibrium transition and the long run when consumers are heterogeneous. In this respect, most papers focus on heterogeneity in wealth (Sorger (2000), Ghiglino and Sorger (2002), Garcia-Penalosa and Turnovsky (2006)).

In this paper, we are interested in the effects of endogenous labor supply on the saddle-path stability when consumers' heterogeneity concerns not only their endowments but also their preferences. In this connection, we consider infinite-lived consumers with preferences which are additively separable over time, but depend on consumption and leisure at each period. Heterogeneity is now threefold and turns on capital wealth, time preference and intra-temporal preferences. In addition, in line with Becker (1980), Becker and Foias (1987, 1994), Hernandez (1991) and Sorger (1994), we assume that consumers cannot borrow against their future labor income. This borrowing constraint implicitly

\footnotetext{
${ }^{1}$ Barro and Sala-i-Martin (1995) provide an introductory but representative survey of the literature.
} 
means that, in contrast to Le Van, Manh Hung and Vailakis (2006), markets are incomplete.

Under these assumptions, we show that, as in Becker (1980), Becker and Foias (1987, 1994), and Sorger (1994), the most patient household owns the whole capital stock in a neighborhood of the steady state, whereas the others consume their per-period labor income. In the rest of the paper, since we are mainly interested in local dynamics around the steady state, we can focus directly on such equilibria with extreme distribution of capital.

Under usual hypotheses, we prove the existence of a unique steady state in contrast with Sorger (2000) who obtains a continuum of long-run equilibria. Sorger's assumption of a common discount factor across the agents prevents a long-run concentration of wealth in the hands of the most patient individual. Survival of many capital owners in the long run entails also a sensitivity of the steady state to disaggregate initial conditions, namely the initial distribution of wealth, accounting for a continuum of stationary equilibria.

When there are no impatient agents in the economy, our model gets closer to De Hek (1998) with a representative agent. However, normality assumptions on consumption and leisure prevents us to obtain multiple steady states in contrast with him, who obtains multiplicity under very unusual conditions.

In order to study the convergence to the (unique) steady state, while underlining the role of heterogeneous preferences and elastic labor supply, we focus on their effects on the saddle-path stability and the occurrence of a flip bifurcation. In economies with inelastic labor supply (Becker and Foias (1994)), the occurrence of endogenous cycles requires sufficiently weak capital-labor substitution. Introducing leisure in the utility functions, we find that the impatient agent's preferences play a role on the stability properties of the steady state.

We derive conditions for saddle-path stability and the occurrence of twoperiod cycles under very general preferences in consumption and leisure. Then, in order to provide a more explicit characterization and to check the robustness of saddle-path stability, we focus on four particular cases.

First, the representative agent's case is nested in the general model and deserves some comparative comment with the existing literature. Second, heterogeneous preferences are considered, but separable in consumption and leisure, in order to simplify and clarify conditions for cycles. Third, a $\mathrm{KPR}^{2}$ specification is taken into account to prove that an elastic labor supply promotes stability. Eventually, the case of homogeneous preferences confirms how robust saddle-path stability is.

In the first case (representative (patient) agent), we gets closer to De Hek (1998). Under very usual restrictions, such as normality, we find a unique capital path, which prevents the economy from deterministic fluctuations and, shortly, we stress his lack of robustness.

In the other cases, heterogeneity is restored. When preferences are separable in consumption and leisure, the impatient agents' intertemporal substitution in consumption matters. More precisely, when their elasticity of intertemporal

\footnotetext{
${ }^{2}$ See King, Plosser and Rebelo (1988).
} 
substitution becomes greater than one, the introduction of elastic labor supplies promotes stability by making conditions for cycles more demanding in terms of technological parameters. However, the opposite conclusion holds when elasticity of intertemporal substitution in consumption is less than one.

The class KPR of utility functions is used in growth literature to lighten restrictions on intra-temporal preferences in order to get positive growth rates. In this case, the patient agent's preferences matter, while the impatient agents' ones play no role on dynamics. More precisely, convergence to the steady state requires an elasticity of intertemporal substitution in consumption not too weak and a leisure utility not too concave, whatever the elasticity of capital-labor substitution.

Finally, when preferences are homogeneous, the patient agent's degree of homogeneity becomes a key parameter. We prove that when it is sufficiently close to one (including the CES and the Cobb-Douglas cases), we recover the saddle-path stability, whatever shape the other fundamentals have.

To understand why preferences play a role on stability properties, we mind that, when labor supply is inelastic, instability ensues from a negative response of the patient agent's income to a rise in the capital stock. When labor supplies become elastic, they also affect his income. In addition, the endogenous labor supplies vary because an increase in the capital stock raises the real wage. Since preferences underlie and determine the elasticities of labor supply with respect to the real wage, unsurprisingly they have a great influence on dynamics.

The rest of the paper is organized as follows. The general model with heterogeneous consumers, elastic labor and borrowing constraints is presented in Section 2. Section 3 is devoted to compute a two-dimensional system representing the equilibrium dynamics. Existence and uniqueness of the steady state are proved in Section 4. Section 5 focuses on the stability properties and the occurrence of bifurcations under unspecified preferences. Sections 6 characterizes an economy with representative consumer. Sections 7 to 9 revisit the general findings in the case of separable, KPR or homogeneous preferences, respectively. In Section 10, we provide an economic interpretation of the results, keeping in mind the stability issue. Section 11 concludes, while technical details are gathered in the Appendix.

\section{A growth model with heterogeneous households}

We address the saddle-path stability issue in a discrete time growth model with heterogeneous agents and borrowing constraints. Consumers are differently endowed with capital and have different preferences. In this respect, we assume a twofold kind of heterogeneity in tastes: on the one hand, heterogeneous discount factors; on the other hand, different instantaneous utilities in consumption and leisure across the households. 


\subsection{Consumers}

There is a finite number $(1+n)$ of heterogeneous infinite lived agents, who are identified by a progressive label $i=0,1, \ldots, n$ according to their time preference, that is, to the (decreasing) ranking of their discount factors:

$$
0 \leq \beta_{n} \leq \ldots \leq \beta_{1}<\beta_{0}<1
$$

We notice that a degree of heterogeneity between the more patient agent $(i=0)$ and the others is required at least.

At each period, the consumer $i$ is endowed with one unit of time that he shares between labor and leisure. We denote his consumption and labor supply at period $t$ with $c_{i t}$ and $l_{i t}$. Preferences are represented by an utility function in consumption and leisure which is separable over time:

$$
\sum_{t=0}^{\infty} \beta_{i}^{t} u_{i}\left(c_{i t}, 1-l_{i t}\right)
$$

Consumer $i$ maximizes (2) under a sequence of budget constraints:

$$
c_{i t}+k_{i t+1}-\Delta k_{i t} \leq r_{t} k_{i t}+w_{t} l_{i t}
$$

and a sequence of borrowing constraints:

$$
k_{i t} \geq 0
$$

Notation is quite usual: $r$ and $w$ denote the interest rate and the wage, respectively, while $\Delta \equiv 1-\delta$ with $\delta \in(0,1)$ the depreciation rate of capital. The initial (heterogeneous) endowments $k_{i 0} \geq 0$ are given, with at least one strictly positive.

Assumption 1 The utility function $u_{i}\left(c_{i}, 1-l_{i}\right)$ is defined on $[0,+\infty) \times$ $[0,1]$ for every $i=0, \ldots, n$; it is $C^{2}$ on $(0,+\infty) \times(0,1)$, increasing in each argument and concave. ${ }^{3}$

$$
\begin{aligned}
u_{i 11} & <0<u_{i 1} \\
u_{i 22} & <0<u_{i 2} \\
u_{i 12} u_{i 21} & \leq u_{i 11} u_{i 22}
\end{aligned}
$$

and satisfies the boundary conditions $\lim _{c_{i} \rightarrow 0} u_{i 1}\left(c_{i}, 1-l_{i}\right) / u_{i 2}\left(c_{i}, 1-l_{i}\right)=$ $+\infty, \lim _{l_{i} \rightarrow 1} u_{i 1}\left(c_{i}, 1-l_{i}\right) / u_{i 2}\left(c_{i}, 1-l_{i}\right)=0$ on $(0,+\infty) \times(0,1)$.

We also introduce a kind of normality between consumption and leisure.

\section{Assumption 2}

$$
u_{i 11} / u_{i 1}<u_{i 21} / u_{i 2} \text { and } u_{i 22} / u_{i 2}<u_{i 12} / u_{i 1}
$$

\footnotetext{
${ }^{3}$ In the following, we set $u_{i j} \equiv \partial u_{i}\left(x_{1}, x_{2}\right) / \partial x_{j}$ for $j=1,2$ and $u_{i j k} \equiv \partial u_{i j}\left(x_{1}, x_{2}\right) / \partial x_{k}$ for $k=1,2$.
} 
We observe that a more usual definition of normality is entailed by inequalities (8) jointly with the consumption-leisure arbitrage $\left(u_{i 2}=w u_{i 1}\right)$ as equilibrium condition in Proposition 1 below.

As intuition suggests and economic literature confirms, ${ }^{4}$ the patient agents will hold the whole stock of capital near the steady state. In order to characterize the equilibrium with high concentration of capital, we need a formal statement.

Proposition 1 Near a steady state, the impatient agents $(i=1, \ldots, n)$ end up with no capital $\left(k_{i t}=0\right)$, while the patient one $(i=0)$ ends up to hold the entire amount of capital $\left(k_{0 t}>0\right)$.

On the one hand, a consumption-leisure arbitrage condition holds for every agent including the patient one $(i=0,1, \ldots, n)$ :

$$
u_{i 2}\left(c_{i t}, 1-l_{i t}\right)=u_{i 1}\left(c_{i t}, 1-l_{i t}\right) w_{t}
$$

On the other hand, the patient capitalist $(i=0)$ and the impatient agents $(i \geq 1)$ have different sequences of budget constraints:

$$
\begin{aligned}
c_{0 t}+k_{0 t+1} & =R_{t} k_{0 t}+w_{t} l_{0 t} \\
c_{i t} & =w_{t} l_{i t}, \text { for } i=1, \ldots, n
\end{aligned}
$$

and smooth consumption differently over time: the capitalist according to a Euler equation:

$$
\frac{u_{01}\left(c_{0 t}, 1-l_{0 t}\right)}{u_{01}\left(c_{0 t+1}, 1-l_{0 t+1}\right)}=\beta_{0} R_{t+1}
$$

whereas the impatient consumers according to the following inequality:

$$
\frac{u_{i 1}\left(w_{t} l_{i t}, 1-l_{i t}\right)}{u_{i 1}\left(w_{t+1} l_{i t+1}, 1-l_{i t+1}\right)}>\beta_{i} R_{t+1}
$$

Individual transversality conditions are also satisfied. ${ }^{5}$

Proof. See the Appendix.

As equation (12) suggests, the patient consumer's discount factor plays a great role in determining capital accumulation. To lighten notation, we will set from now on $\beta \equiv \beta_{0}$ and refer equivalently to the more patient agent as "the capitalist".

For the sake of conciseness, we introduce also the elasticities of marginal utility through which slope and concavity of the utility functions will be characterized:

$$
\left[\begin{array}{ll}
\varepsilon_{i 11} & \varepsilon_{i 12} \\
\varepsilon_{i 21} & \varepsilon_{i 22}
\end{array}\right] \equiv\left[\begin{array}{ll}
\frac{u_{i 11} c_{i}}{u_{i 1}} & \frac{u_{i 12}\left(1-l_{i}\right)}{u_{i 1}} \\
\frac{u_{i 21} c_{i}}{u_{i 2}} & \frac{u_{i 22}\left(1-l_{i}\right)}{u_{i 2}}
\end{array}\right]
$$

\footnotetext{
${ }^{4}$ Among others, the reader is referred to the seminal work of Becker (1980).

${ }^{5}$ Since $k_{i t+1} \rightarrow 0$ for every $i \geq 1$, what actually matters is the capitalist's transversality condition:

$$
\lim _{t \rightarrow+\infty} \beta^{t} u_{01}\left(c_{0 t}, 1-l_{0 t}\right) k_{t+1}=0
$$


We notice that the concavity restrictions (5) and (6) (on the left-hand-side) and (7) now become:

$$
\varepsilon_{i 11}<0, \varepsilon_{i 22}<0 \text { and } \varepsilon_{i 11} \varepsilon_{i 22} \geq \varepsilon_{i 12} \varepsilon_{i 21}
$$

for $i=0,1, \ldots, n$, respectively. Normality inequalities (8) (Assumption 2) also simplify:

$$
\varepsilon_{i 11}<\varepsilon_{i 21} \text { and } \varepsilon_{i 22}<\varepsilon_{i 12}
$$

for $i=0,1, \ldots, n$.

\section{$2.2 \quad$ Firms}

We denote the aggregate capital and labor with $k_{t}$ and $l_{t}$, respectively. The representative firm produces the final good $y_{t}=F\left(k_{t}, l_{t}\right) \equiv f\left(a_{t}\right) l_{t}$ with a constant returns to scale technology and a degree of capital intensity $a_{t} \equiv k_{t} / l_{t}$, in order to maximize the profit $\pi_{t} \equiv y_{t}-r_{t} k_{t}-w_{t} l_{t}$ at every period $t$. The intensive production function $f$ satisfies standard neoclassical requirements.

Assumption 3 The intensive production $f(a)$ is a continuous function of the capital-labor ratio $a \geq 0$, positive-valued and differentiable, as many times as needed, for $a>0$, with $f^{\prime \prime}(a)<0<f^{\prime}(a)$ and $f(0)=0, \lim _{a \rightarrow 0} f^{\prime}(a)=+\infty$, $\lim _{a \rightarrow+\infty} f^{\prime}(a)=0$.

Firms' profit maximizing behavior entails that production takes place in period $t$ so that the capital-labor ratio $a_{t}$ equates the real rental rate of capital services $r_{t}$ with the marginal productivity of capital and the real wage $w_{t}$ with the marginal productivity of labor.

$$
\begin{aligned}
r_{t} & =f^{\prime}\left(a_{t}\right) \equiv r\left(a_{t}\right) \\
w_{t} & =f\left(a_{t}\right)-f^{\prime}\left(a_{t}\right) a_{t} \equiv w\left(a_{t}\right)
\end{aligned}
$$

It is not unworthy to highlight the underlying relations existing between the elasticities of the interest rate with respect to capital and labor $r_{k} k / r$, $r_{l} l / r$, the analogous elasticities of the real wage $w_{k} k / w, w_{l} l / w$, the capital share in total income $s(a) \equiv f^{\prime}(a) a / f(a) \in(0,1)$ and the elasticity of capitallabor substitution: $\sigma(a) \equiv\left[a f^{\prime}(a) / f(a)-1\right] f^{\prime}(a) /\left[a f^{\prime \prime}(a)\right]>0$. Standard computations give $k r_{k} / r=-l r_{l} / r=-[1-s(a)] / \sigma(a)$ and $k w_{k} / w=-l w_{l} / w=$ $s(a) / \sigma(a)$.

\subsection{Equilibrium}

An intertemporal equilibrium is a sequence of prices (or, dually, of quantities) that clears the markets in every period. Equilibria in good and labor markets require:

$$
c_{t}+k_{t+1}-\Delta k_{t}=F\left(k_{t}, l_{t}\right) \text { and } l_{t}=\sum_{i=0}^{n} l_{i t} \text {, }
$$


where $c_{t} \equiv \sum_{i=0}^{n} c_{i t}$ denotes the aggregate consumption, for $t=0,1, \ldots,+\infty$. We observe that equation (20) is immediately obtained by aggregating budget constraints (10) and (11) across the individuals. Since the patient agent ends up to hold all the capital stock, we have also the following condition:

$$
k_{t}=k_{0 t}
$$

In order to find the equilibrium path prevailing around the steady state and characterized by a concentration of capital in the hands of a single agent (equation (21)), we derive the dynamic system and study the local dynamics around its stationary solution.

\section{Dynamic system}

Equation (9) is informative about the slope of the patient agent's labor supply $l_{0}$. We keep (9) with $i=0$ and we solve (locally) for $l_{0}$ :

$$
l_{0 t}=l_{0}\left(w_{t}, c_{0 t}\right)
$$

Substituting (11) in (9), we transform the consumption-arbitrage condition of the $i$ th impatient agent in an implicit equation relating his labor supply to the real wage:

$$
u_{i 2}\left(w_{t} l_{i t}, 1-l_{i t}\right)=u_{i 1}\left(w_{t} l_{i t}, 1-l_{i t}\right) w_{t}
$$

or, locally,

$$
l_{i t}=l_{i}^{*}\left(w_{t}\right)
$$

We observe that the labor supply of an impatient agent just depends on the real wage, while the patient agent's labor supply depends also on his consumption demand. To ensure that labor supplies are correctly defined as functions, we use the assumptions made above on the shapes of utility and production functions.

Lemma 2 If $\lim _{l_{0 t} \rightarrow 0} u_{02}\left(c_{0 t}, 1-l_{0 t}\right) / u_{01}\left(c_{0 t}, 1-l_{0 t}\right)<w_{t}$ and Assumptions 1-3 are satisfied, the functions $l_{i t}=l_{i}^{*}\left(w_{t}\right)$ and $l_{0 t}=l_{0}\left(c_{0 t}, w_{t}\right)$ are well defined.

Proof. See the Appendix.

Since the purpose of the section is deriving a dynamic system, we need to reduce the number of variables, aggregating, for instance, the labor supplies. In this respect, putting together equations (19), (20), (22) and (24), we obtain:

$$
l_{t}=l_{0}\left(w\left(k_{t} / l_{t}\right), c_{0 t}\right)+\sum_{i=1}^{n} l_{i}^{*}\left(w\left(k_{t} / l_{t}\right)\right)
$$

that is an implicit equation relating the aggregate labor supply $l$ to the aggregate capital $k$ and the individual consumption $c_{0}$. More explicitly, but locally under the usual hypotheses of the implicit function theorem, we get:

$$
l_{t} \equiv l\left(k_{t}, c_{0 t}\right)
$$


At the very core of dynamics is the capitalist's behavior: Replacing expressions (18), (19), (22) and (26) in his budget constraint (10) and in his Euler equation (12), eventually, we obtain the equilibrium dynamics:

$$
\begin{aligned}
& \frac{u_{01}\left(c_{0 t}, 1-l_{0}\left(w\left(k_{t} / l\left(k_{t}, c_{0 t}\right)\right), c_{0 t}\right)\right)}{u_{01}\left(c_{0 t+1}, 1-l_{0}\left(w\left(k_{t+1} / l\left(k_{t+1}, c_{0 t+1}\right)\right), c_{0 t+1}\right)\right)}=\beta\left[\Delta+r\left(\frac{k_{t+1}}{l\left(k_{t+1}, c_{0 t+1}\right)}\right)\right] \\
& c_{0 t}+k_{t+1}-\Delta k_{t}=r\left(\frac{k_{t}}{l\left(k_{t}, c_{0 t}\right)}\right) k_{t}+w\left(\frac{k_{t}}{l\left(k_{t}, c_{0 t}\right)}\right) l_{0}\left(w\left(\frac{k_{t}}{l\left(k_{t}, c_{0 t}\right)}\right), c_{0 t}\right)
\end{aligned}
$$

where the initial condition $k_{0}>0$ is given ${ }^{6}$ and the transversality condition (14) holds.

Dynamic system (27)-(28) deserves some comments. It holds at every period, $t=0,1, \ldots,+\infty$, and defines an intertemporal sequence $\left(c_{0 t}, k_{t}\right)_{t>0}$. Usually, the dynamic system of the Ramsey model is written in the representative agent's pair of variables $(k, c)$. The capital stock $k$ is a predetermined state variable, while the consumption expenditure $c$ jumps in order to make the equilibrium path positive and compatible with the transversality condition. In our model, the aggregate capital remains predetermined, but the aggregate consumption is replaced by the disaggregate capitalist's consumption. ${ }^{7}$

In the following, first, the uniqueness of the steady state will be stated; then, saddle-path stability and the existence of flip and period-doubling bifurcations will be characterized through the analysis of a linear approximation of system (27)-(28) around the steady state.

\section{Steady state}

A stationary state of dynamic system $(27)-(28)$ is a solution $\left(k, c_{0}\right)=\left(k_{t}, c_{0 t}\right)$ of the following system for all $t=0,1, \ldots,+\infty$ :

$$
\begin{aligned}
\frac{1}{\beta}-\Delta & =r\left(\frac{k}{l\left(k, c_{0}\right)}\right) \\
c_{0} & =\frac{1-\beta}{\beta} k+w\left(\frac{k}{l\left(k, c_{0}\right)}\right) l_{0}\left(w\left(\frac{k}{l\left(k, c_{0}\right)}\right), c_{0}\right)
\end{aligned}
$$

where the functions $l_{0}\left(w, c_{0}\right)$ and $l\left(k, c_{0}\right)$ are now defined at the steady state by (22) and (26), respectively. In the next proposition, we analyze the existence and the uniqueness of such stationary solution:

Proposition 3 Under Assumptions 1-3, there exists a unique steady state.

\footnotetext{
${ }^{6}$ The initial aggregate capital stock is defined by $k_{0}=\sum_{i=0}^{n} k_{i 0}$. The equilibria considered in this section implicitly requires an initial distribution of capital close enough to that of Proposition 1.

${ }^{7}$ Writing an equivalent system in the pair of variables $(k, c)$, where $c$ is the aggregate consumption, or in the pair $(k, l)$, will complicate needlessly the size of equations under study, whereas the associated eigenvalues are invariant to the choice of variables.
} 
Proof. See the Appendix.

In contrast to Sorger (2000), there is no room for a continuum of stationary solutions parametrized by the initial distribution of wealth. In Sorger's paper, agents have heterogeneous initial capital endowments, but share the same preferences and in particular the same discount factor. Since in our case the more patient agent ends up to hold the whole capital stock, the stationary distribution of wealth is determined by his heterogeneous discount factor and the existence of borrowing constraints, and does not meet Sorger's conditions for multiplicity.

Eventually, we notice that, setting $n=0$ and $l_{t}=l_{0}\left(w_{t}, c_{0 t}\right)$, we get the optimal growth model with representative consumer as particular case. In this respect, Proposition 3 still applies. Our conventional assumptions entail results which appear in open contrast with those obtained by De Hek (1998) who analyzes an aggregate optimal growth model with leisure-depending preferences. In order to reconcile the papers, we simply observe that his multiplicity of steady states rest on some unusual assumptions, namely the lack of normality between consumption and leisure. ${ }^{8}$

\section{On the saddle-path stability}

Convergence to the steady state is the core of the paper. This issue is addressed through a local analysis, i.e., through the analysis of a neighborhood of the steady state, where the intertemporal equilibrium defined in Proposition 1 precisely holds.

In order to characterize the stability properties of the steady state and the occurrence of local bifurcations, we proceed by linearizing the dynamic system (27)-(28) around the steady state $\left(k, c_{0}\right)$ and computing the Jacobian matrix $J$, evaluated at this steady state. Local dynamics are represented by a linear system $\left(d k_{t+1} / k, d c_{0 t+1} / c_{0}\right)^{T}=J\left(d k_{t} / k, d c_{0 t} / c_{0}\right)^{T}$. In the following, we exploit the fact that the trace $T$ and the determinant $D$ of $J$ are the sum and the product of the eigenvalues, respectively. As emphasized by Grandmont, Pintus and de Vilder (1998), the stability properties of the system, that is, the location of the eigenvalues with respect to the unit circle, can be better characterized in the $(T, D)$-plane (see Figures 1-3).

In concrete terms, we evaluate the characteristic polynomial $P(\alpha) \equiv \alpha^{2}-$ $T \alpha+D$ at $-1,0,1$. Along the line $(A C)$, one eigenvalue is equal to 1 , i.e., $P(1)=1-T+D=0$. Along the line $(A B)$, one eigenvalue is equal to -1 , i.e., $P(-1)=1+T+D=0$. On the segment $[B C]$, the two eigenvalues are complex and conjugate with unit modulus, i.e., $D=1$ and $|T|<2$. Therefore, inside the triangle $A B C$, the steady state is a sink, i.e., locally indeterminate $(D<1$ and $|T|<1+D)$. It is a saddle point if $(T, D)$ lies on the right sides of both $(A B)$ and $(A C)$ or on the left sides of both of them $(|1+D|<|T|)$. It is a source otherwise. A (local) bifurcation arises when an eigenvalue crosses

\footnotetext{
${ }^{8}$ In Section 6, the unconventional equilibrium transitions found by De Hek (1998) are questioned on the same basis.
} 
the unit circle, that is, when the pair $(T, D)$ crosses one of the loci $(A B)$, $(A C)$ or $[B C]$. $(T, D)$ depends on the structural parameters. We choose a parameter of interest and we study how $(T, D)$ moves with it in the $(T, D)$ plane. More explicitly, according to the changes in the bifurcation parameter, a transcritical bifurcation (generically) occurs when $(T, D)$ goes through $(A C)$, a flip bifurcation (generically) arises when $(T, D)$ crosses $(A B)$, whereas a Hopf bifurcation (generically) emerges when $(T, D)$ goes through the segment $[B C]$.

The expressions of $T$ and $D$ involve the elasticities of labor supply, which are a central link in the chain of effects accounting for the degree of stability. From (22) and (24), one defines these elasticities as $\xi_{0} \equiv\left(\partial l_{0} / \partial w\right)\left(w / l_{0}\right)$ for the capitalist, $\xi_{i} \equiv l_{i}^{* \prime}(w) w / l_{i}$ for the impatient agents. The average elasticity of labor supply $\xi \equiv \sum_{i=0}^{n} \lambda_{i} \xi_{i}$ is naturally defined, by weighting the individual elasticities with the individual labor shares in total labor supply $\lambda_{i} \equiv l_{i} / l_{i} i=0,1, \ldots, n .^{9}$ Another average elasticity of labor supply of interest is $\tilde{\xi} \equiv \sum_{i=1}^{n} \lambda_{i} \xi_{i} /\left(1-\lambda_{0}\right)$, which takes in account only the impatient agents' elasticities $\xi_{i}$ 's and summarizes the role of their preferences on the dynamics. Clearly, $\xi=\lambda_{0} \xi_{0}+\left(1-\lambda_{0}\right) \tilde{\xi}$. We also denote the elasticity of capitallabor substitution with $\sigma$ and the capital share in total income evaluated at the steady state with $s$. Eventually, we need to introduce a reduced parameter $\rho \equiv \beta r(1-s) / s=(1-\beta \Delta)(1-s) / s$, before presenting the determinant and the trace: ${ }^{10}$

$$
\begin{aligned}
D(\sigma) & =\frac{1}{\beta}-\frac{\rho}{\beta} \frac{1}{\tilde{\xi}-\tilde{\xi}^{*}+\frac{\sigma}{s} \frac{1}{1-\lambda_{0}}} \\
T(\sigma) & =1+\frac{1}{\beta}-\frac{\rho}{\beta} \frac{1}{\tilde{\xi}-\tilde{\xi}^{*}+\frac{\sigma}{s} \frac{1}{1-\lambda_{0}}} \frac{1}{S}
\end{aligned}
$$

where

$$
\begin{aligned}
\tilde{\xi}^{*} & \equiv \frac{1-l_{0}}{l-l_{0}} \frac{\varepsilon_{011}+\rho\left(\varepsilon_{011}-\varepsilon_{021}\right)}{\varepsilon_{011} \varepsilon_{022}-\varepsilon_{012} \varepsilon_{021}}<0 \\
S & \equiv\left[1-\frac{(1-\beta+\rho)\left(\varepsilon_{021}-\varepsilon_{011}\right)\left(1-l_{0}\right)+\left(1-\beta+\rho \lambda_{0}\right)\left(\varepsilon_{012}-\varepsilon_{022}\right) l}{\left(\varepsilon_{011} \varepsilon_{022}-\varepsilon_{012} \varepsilon_{021}\right)\left(l-l_{0}\right)}\right]^{-1}
\end{aligned}
$$

In order to apply the geometrical method introduced by Grandmont, Pintus and de Vilder (1998), we need to choose carefully the parameter with respect to which the bifurcation analysis is leaded. An ideal choice is a significant parameters from an economic point of view, which has, in addition, a linear image in the $(T, D)$-plane: a half-line is unambiguously determined by the origin and the slope. Fortunately, the elasticity of capital-labor substitution $\sigma$ meets both the criteria: ${ }^{11}$ The locus $\Sigma \equiv\{(T(\sigma), D(\sigma)): \sigma \geq 0\}$ is either a (connected)

\footnotetext{
${ }^{9}$ Notice also that $\sum_{i=0}^{n} \lambda_{i}=1$.

${ }^{10}$ The detailed computations of $J, T$ and $D$ are relegated in the Appendix.

${ }^{11}$ In a Ramsey model with heterogeneous consumers and borrowing constraints, but inelastic labor supplies, Becker and Foias (1994) choose the same parameter to make the (flip) bifurcation analysis, while following a different (non-geometrical) approach.
} 
segment or an (unconnected) half-line in the (T, D)-plane (see Figures 1-3) with an origin $(\sigma=0)$ :

$$
\begin{aligned}
& D(0)=\frac{1}{\beta}-\frac{\rho}{\beta} \frac{1}{\tilde{\xi}-\tilde{\xi}^{*}} \\
& T(0)=1+\frac{1}{\beta}-\frac{\rho}{\beta} \frac{1}{\tilde{\xi}-\tilde{\xi}^{*}} \frac{1}{S}
\end{aligned}
$$

an endpoint $(\sigma=+\infty)$ :

$$
(T(+\infty), D(+\infty))=(1+1 / \beta, 1 / \beta)
$$

and a slope $D^{\prime}(\sigma) / T^{\prime}(\sigma)=S$. From (33), we note that $S \notin[0,1]$. Moreover, the endpoint $\left(T_{1}(+\infty), D_{1}(+\infty)\right)$ is on the line $(A C)$, above the point $C$.

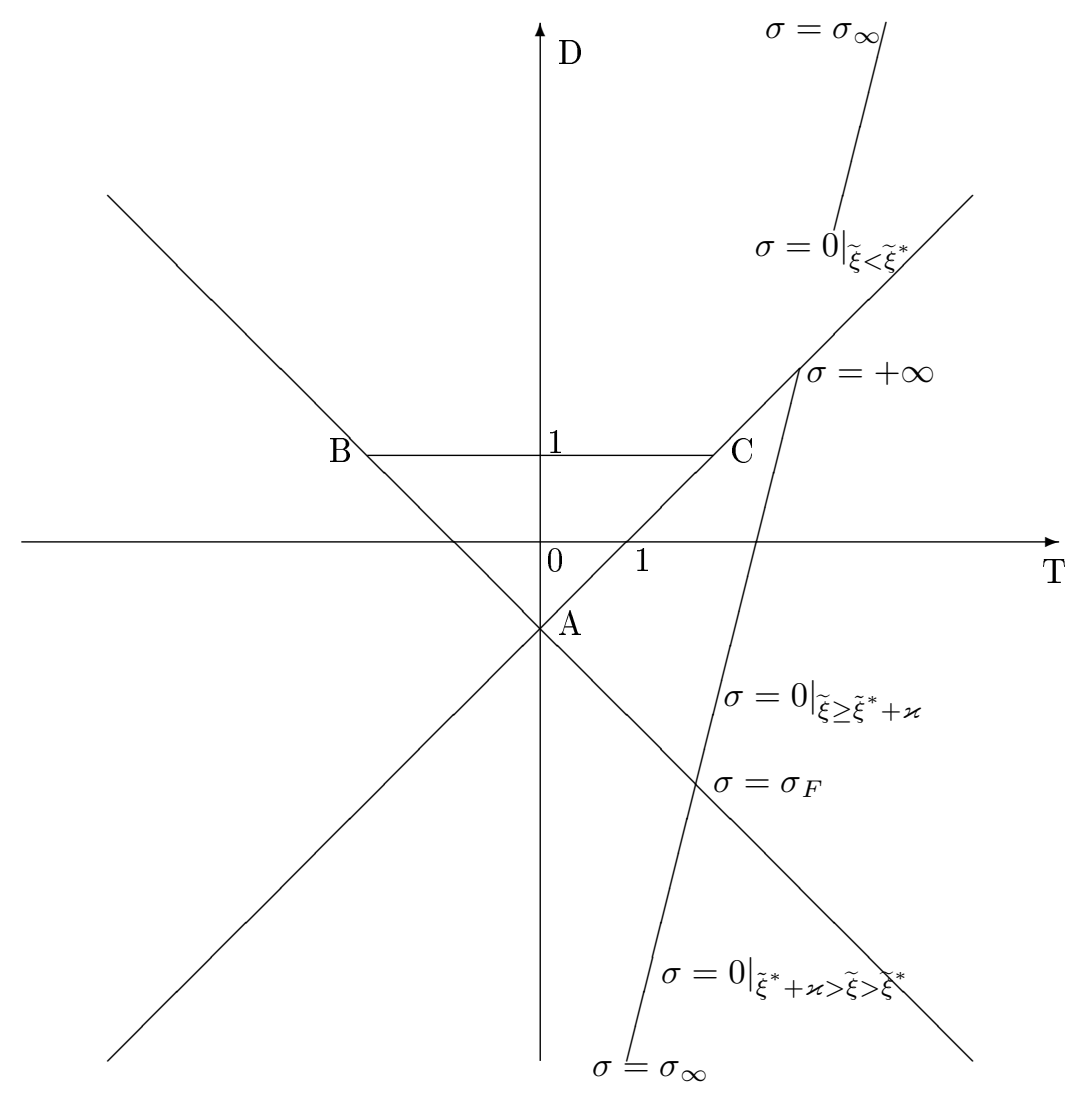

Figure 1: $S>1$

The locus $\Sigma$ is either a segment or a half-line. Even if the line including $\Sigma$ always crosses the line $(A B),{ }^{12}$ the intersection of $(A B)$ with $\Sigma$ could be

${ }^{12}$ The case $S=-1$ is generically omitted. 


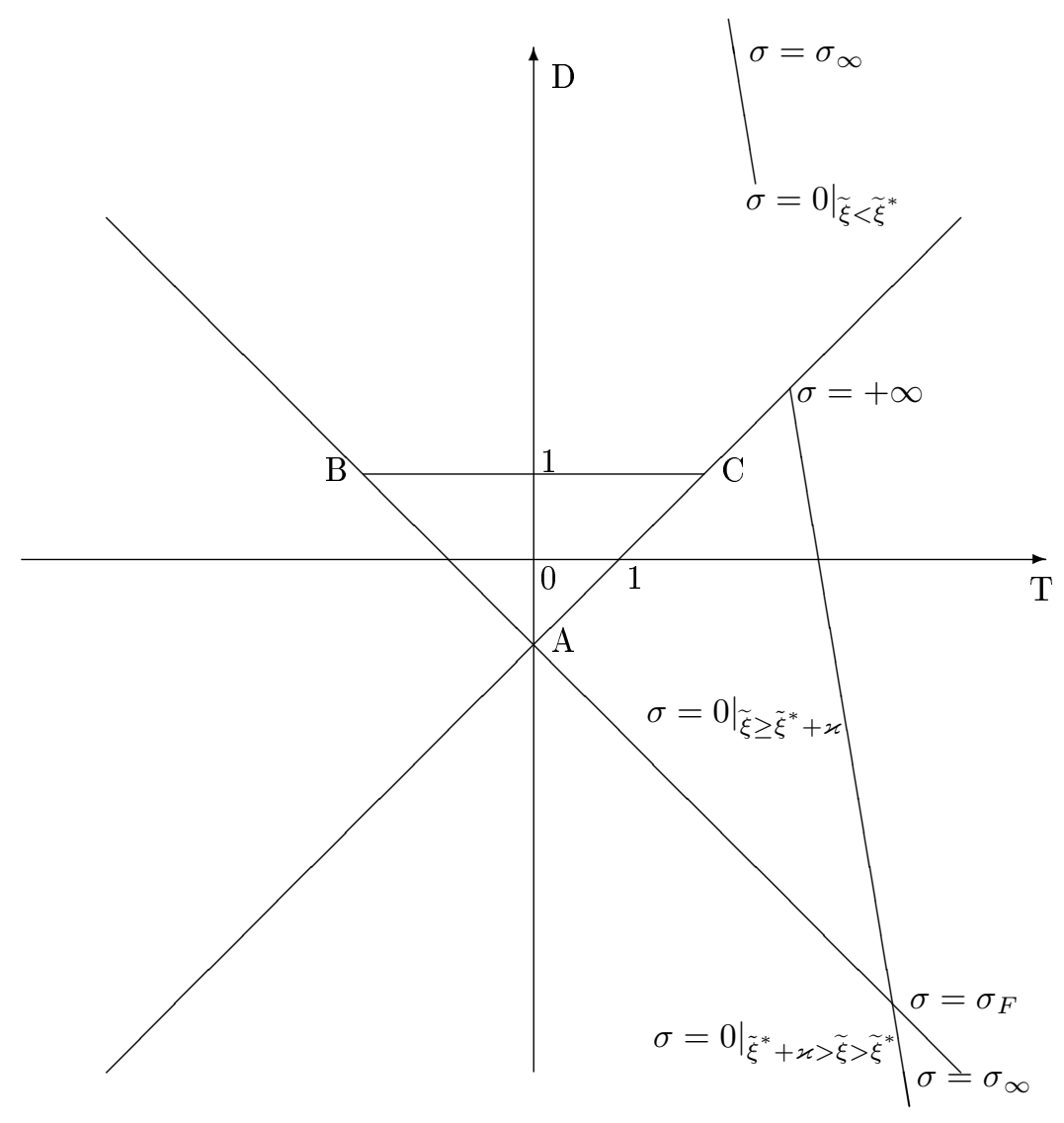

Figure 2: $S<-1$

empty. In order to characterize the shape of $\Sigma$ and provide clear-cut bifurcation conditions, we need to detail the properties of trace and determinant as functions of $\sigma$.

Since $D^{\prime}(\sigma)>0$, when $\sigma$ increases from 0 to $+\infty$, the point $(T(\sigma), D(\sigma))$ moves always upward along $\Sigma$ from the origin $(T(0), D(0))$, to the endpoint $(T(+\infty), D(+\infty))$. If $\Sigma$ crosses the line $(A B)$, then a flip bifurcation generically occurs for a positive value of $\sigma$, say $\sigma_{F}$, corresponding to the intersection and solving the equation $D(\sigma)=-T(\sigma)-1$.

A quick look to the $(T, D)$-plane will convince the reader that few informations are required to locate $\Sigma$ and determine the stability properties of the steady state.

(1) Is the origin $(T(0), D(0))$ above or below the endpoint $(1+1 / \beta, 1 / \beta)$ ?

(2) Is the origin $(T(0), D(0))$ above or below the flip line $(A B)$ ?

(3) Is the slope steeper $(|S|>1)$ or flatter $(-1<S<0)$ ? 


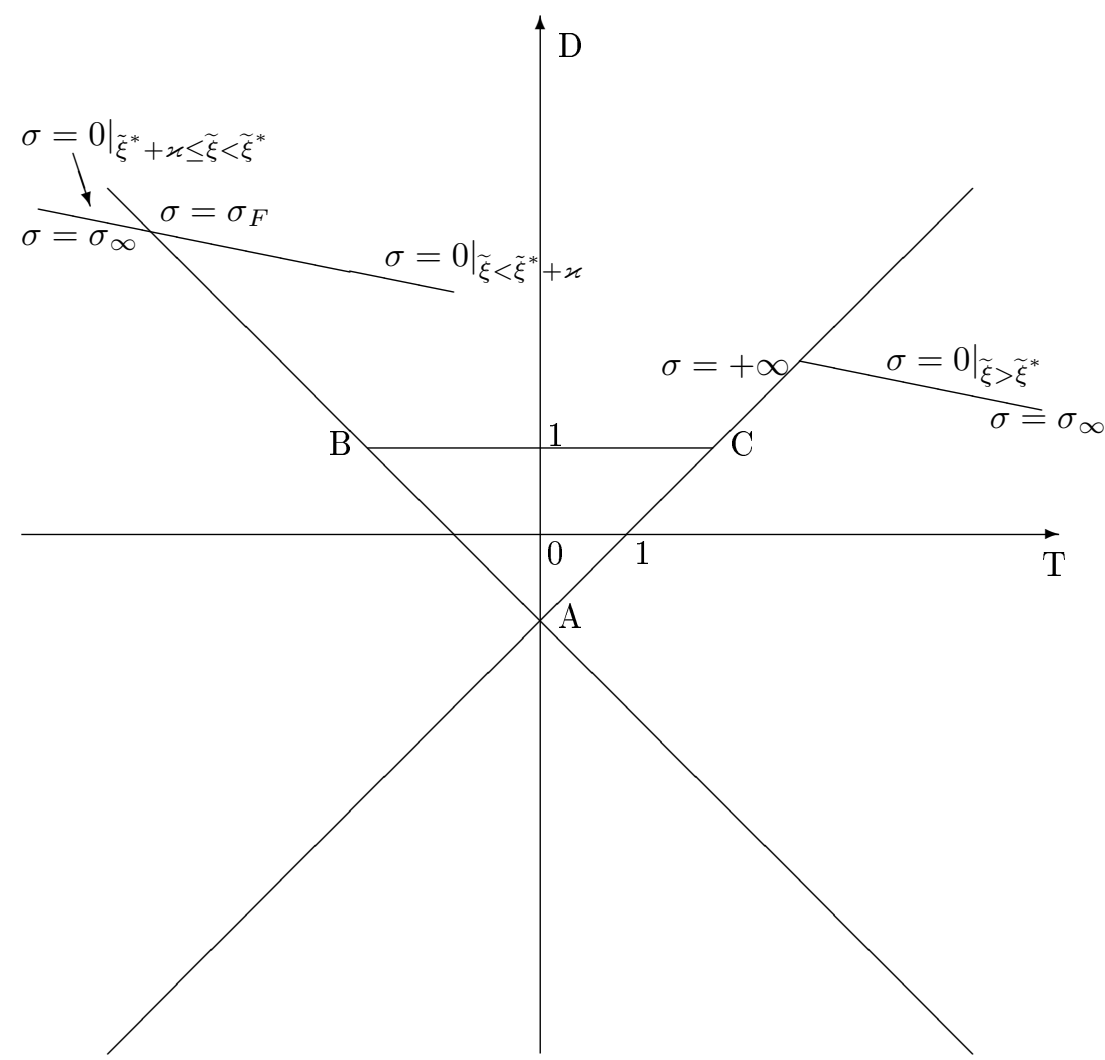

Figure 3: $-1<S<0$

We first find explicit conditions for points (1) and (2).

(1) The necessary and sufficient condition for the origin to stay above the endpoint is $D(0)>1 / \beta$, that is

$$
\tilde{\xi}<\tilde{\xi}^{*}
$$

In this case, $\Sigma$ is a half-line starting from $D=D(0)>1 / \beta$, going up to $D=+\infty$ and jumping to $D=-\infty$ for $\sigma=\sigma_{\infty}$, and going up to $D=1 / \beta$, with $\sigma_{\infty} \equiv s\left(1-\lambda_{0}\right)\left(\tilde{\xi}^{*}-\tilde{\xi}\right)$.

(2) The necessary and sufficient condition for the origin $(T(0), D(0))$ to lie above the line $(A B)$ is $D(0)>-T(0)-1$ or, equivalently,

$$
\varkappa /\left(\tilde{\xi}-\tilde{\xi}^{*}\right)<1
$$

where

$$
\varkappa \equiv \rho(1+S) /[2(1+\beta) S]
$$


Using these two results, we discuss now the stability properties of the steady state and the occurrence of bifurcations considering two cases for the slope.

(3) We assume $|S|>1$ and $-1<S<0$ in order.

(3.1) $|S|>1: \varkappa$ is positive and three subcases matter (see Figures 1 and 2):

(3.1.1) If $\tilde{\xi}<\tilde{\xi}^{*}$, inequalities (37) and (38) are satisfied. $(T(0), D(0))$ is above the endpoint $(T(+\infty), D(+\infty))$ and the line $(A B) . \Sigma$ is a jumping half-line that crosses the line $(A B)$ at $\sigma=\sigma_{F}$. Therefore, the steady state is a source for $0<\sigma<\sigma_{F}$ and a saddle for $\sigma>\sigma_{F}$.

(3.1.2) If $\tilde{\xi}^{*}<\tilde{\xi}<\tilde{\xi}^{*}+\varkappa$, inequalities (37) and (38) are no longer satisfied. The origin is below the endpoint and the line $(A B) . \Sigma$ is a segment that crosses the line $(A B)$. Therefore, the steady state is a source for $0<\sigma<\sigma_{F}$ and a saddle for $\sigma>\sigma_{F}$.

(3.1.3) If $\tilde{\xi}^{*}+\varkappa<\tilde{\xi}$, inequality (37) is not satisfied, while (38) is. The origin is below the endpoint and above the line $(A B) . \Sigma$ is a segment that does not cross the line $(A B)$, which means that the steady state is a saddle whatever $\sigma$.

(3.2) $-1<S<0$ : $\varkappa$ is negative and three subcases matter (see Figure 3 ):

(3.2.1) If $\tilde{\xi}<\tilde{\xi}^{*}+\varkappa$, inequalities (37) and (38) are satisfied. The origin is above the endpoint and the line $(A B) . \quad \Sigma$ is a jumping half-line that crosses the line $(A B)$. Therefore, the steady state is a source for $0<\sigma<\sigma_{F}$ and a saddle for $\sigma>\sigma_{F}$.

(3.2.2) If $\tilde{\xi}^{*}+\varkappa<\tilde{\xi}<\tilde{\xi}^{*}$, inequality (37) is satisfied, while (38) no longer holds. The origin is above the endpoint, but below the line $(A B) . \Sigma$ is a jumping half-line that does not cross the line $(A B)$. The steady state is a saddle whatever $\sigma$.

(3.2.3) If $\tilde{\xi}^{*}<\tilde{\xi}$, inequality (37) is no longer satisfied, while (38) is. The origin is below the endpoint and above the line $(A B) . \Sigma$ is a segment that does not cross the line $(A B)$. As in the previous subcase, the steady state is a saddle for all $\sigma$.

Summing up, we notice that, when $\tilde{\xi}<\tilde{\xi}^{*}+\varkappa$, the steady state is a source for $0<\sigma<\sigma_{F}$ and a saddle for $\sigma>\sigma_{F}$, and the system undergoes a flip bifurcation at $\sigma=\sigma_{F}$ (subcases (3.1.1), (3.1.2), (3.2.1)). In contrast, when $\tilde{\xi}^{*}+\varkappa<\tilde{\xi}$, we get a saddle, whatever $\sigma$ (subcases $\left.(3.1 .3),(3.2 .2),(3.2 .3)\right)$.

All these necessary and sufficient conditions are summarized in the next proposition.

Proposition 4 Let

$$
\sigma_{F} \equiv s\left(1-\lambda_{0}\right)\left(\tilde{\xi}^{*}+\varkappa-\tilde{\xi}\right)
$$


where $\tilde{\xi}^{*}$ and $\varkappa$ are given by (32) and (39), respectively. The following results generically hold.

If the impatient agents' average elasticity of labor supply is greater than a threshold, that is, $\tilde{\xi} \geq \tilde{\xi}^{*}+\varkappa$, the steady state is a saddle, whatever the degree of capital-labor substitution $\sigma>0$.

If the impatient agents' average elasticity of labor supply is smaller than a threshold, that is, $\tilde{\xi}<\tilde{\xi}^{*}+\varkappa$, the steady state is a source when inputs are rather complementary $\left(0<\sigma<\sigma_{F}\right)$ and it is a saddle when they are rather substitutable $\left(\sigma>\sigma_{F}\right)$. When $\sigma$ crosses $\sigma_{F}$, the system undergoes a flip bifurcation.

This proposition states that the steady state is always locally determinate. However, saddle-path stability and local convergence is not always ensured. When the substitution between capital and labor is sufficiently weak, the steady state can loose stability through the occurrence of a cycle of period two.

Proposition 4 is as general as somewhat cryptic. In order to make the role of preferences on (in)stability more explicit and provide an economic interpretation of bifurcation, we have to go beyond the generality of proposition and specify the fundamentals. Four canonical cases are retained.

At the first stage, we recover the particular case of a representative agent in order to compare our results with De Hek (1998).

At the second stage, we come back to heterogeneous consumers and we analyze three classes of preferences commonly employed in economic literature: separable, $\mathrm{KPR}^{13}$ and homogeneous utility functions in consumption and leisure.

\section{Representative agent}

In order to compare our model to the benchmark, we reduce the number of agents to one, the patient agent. In this case, we are also able to criticize De Hek (1998), who characterize transitional dynamics with representative consumer and elastic labor supply under somewhat unconventional assumptions.

Setting $n=0$, we reduce agents' heterogeneity to the patient agent, now representative. Formally, let $\lambda_{0}=1$ : the average elasticity of labor supply becomes $\xi=\xi_{0}$, while the locus $\Sigma$ becomes a horizontal segment defined by:

$$
\Sigma=\{(T, D): D=1 / \beta, T(+\infty) \leq T \leq T(0)\}
$$

where still $T(+\infty)=1+1 / \beta$, but now

$$
T(0)=1+\frac{1}{\beta}+\frac{\rho(1-\beta+\rho)}{\beta} \frac{\varepsilon_{021}-\varepsilon_{011}+\left(\varepsilon_{012}-\varepsilon_{022}\right) l_{0} /\left(1-l_{0}\right)}{\rho\left(\varepsilon_{021}-\varepsilon_{011}\right)-\varepsilon_{011}}
$$

$\Sigma$ lies entirely on the right-hand side of the endpoint $(1 / \beta, 1+1 / \beta)$ in the region of the saddle points. Indeed, it is enough to observe that the slope $S$ is zero (expression (33)), that $T^{\prime}(\sigma)<0$ (expression (31)) and that $T(0)>$

\footnotetext{
${ }^{13}$ Popularized by King, Plosser and Rebelo (1988), these preferences allow a positive growth rate in endogenous growth models.
} 
$T(+\infty)=1+1 / \beta$, under the standard concavity and normality assumptions (16) and (17).

Let us summarize these findings as follows.

Corollary 5 Without heterogeneity, the steady state is always a saddle and the convergence to the steady state is monotonic.

In contrast to De Hek (1998), we have proved that neither non-monotonic trajectories nor endogenous cycles occur in a one-sector optimal growth model with leisure in the utility function. The key assumption is that of normality (inequalities (17) entailing $T(0)>T(+\infty)$ ). As a matter of fact, De Hek not only introduces a production function which depends on past labor supply and is open to criticism; but also, more questionably, does not impose that consumption and leisure are normal goods at a macroeconomic level.

In the following, we come back to the model with heterogeneous agents and borrowing constraints. As seen above, to understand the role of preferences on saddle-path stability, we specify three classes of utility functions, widely used in economic models: the separable, the KPR and the homogeneous preferences.

\section{Separable utilities}

Assume the utility functions of all consumers to be separable in consumption and leisure, i.e., take the form $u_{i}\left(c_{i t}, 1-l_{i t}\right) \equiv v_{i}\left(c_{i t}\right)+w_{i}\left(1-l_{i t}\right)$, with $v_{i}^{\prime}\left(c_{i}\right)>$ $0, w_{i}^{\prime}\left(1-l_{i}\right)>0, v_{i}^{\prime \prime}\left(c_{i}\right) \leq 0, w_{i}^{\prime \prime}\left(1-l_{i}\right) \leq 0$. Still applying the results of Proposition 4 , we will able to capture the role of concavity in consumption and leisure on convergence.

Separability and concavity of the utility functions of all consumers $i=$ $0,1, \ldots, n$ entail $\varepsilon_{i 12}=\varepsilon_{i 21}=0$ and $\varepsilon_{i 11}<0, \varepsilon_{i 22}<0$, respectively. Then, the critical values for the impatient agents' average elasticity of labor supply and the elasticity of capital-labor substitution, involved in Proposition 4, simplify:

$$
\begin{aligned}
\tilde{\xi}^{*} & \equiv-\frac{1+\rho}{1-\lambda_{0}} \lambda_{0} \xi_{0} \\
\tilde{\xi}^{*}+\varkappa & =\frac{1}{1-\lambda_{0}}\left[\frac{\sigma_{I}}{s}-\lambda_{0} \xi_{0}\left(1+\frac{\rho}{2} \frac{3+\beta+\rho}{1+\beta}\right)\right] \\
\sigma_{F} & \equiv \sigma_{I}-s\left[\left(1-\lambda_{0}\right) \tilde{\xi}+\lambda_{0} \xi_{0}\left(1+\frac{\rho}{2} \frac{3+\beta+\rho}{1+\beta}\right)\right]
\end{aligned}
$$

where $\xi_{0}=-\left(1-l_{0}\right) /\left(l_{0} \varepsilon_{022}\right)$ is now the capitalist's (partial) elasticity of labor supply and:

$$
\sigma_{I} \equiv \frac{s \rho}{1+\beta}\left(1-\lambda_{0}+\frac{1-\beta+\rho \lambda_{0}}{2 \varepsilon_{011}}\right)
$$

It is not unworthy to focus on the limit case with inelastic labor supply, already investigated by Becker and Foias $(1987,1994)$, in order to make ideas clearer about the role of endogenous labor supplies on the stability properties. 


\subsection{Inelastic labor supply}

When agents supply labor inelastically, the leisure elasticity $\varepsilon_{i 22}$ falls to $-\infty$ and, according to formulas (42), (70) and (72), $\xi_{i}=\tilde{\xi}=\tilde{\xi}^{*}=0$ for every $i \geq 0$. By Proposition 4, the occurrence of a flip bifurcation needs $\tilde{\xi}<\tilde{\xi}^{*}+\varkappa$, that is, now, a positive critical elasticity $\sigma_{F}=\sigma_{I}$. In the light of equation (44), the patient consumer is required to have a sufficiently weak intertemporal substitution in consumption, i.e., $-1 / \varepsilon_{011}<2\left(1-\lambda_{0}\right) /\left(1-\beta+\rho \lambda_{0}\right)$.

According to expression (44), the bifurcation value $\sigma_{I}$ decreases in the elasticity of intertemporal substitution in consumption $-1 / \varepsilon_{011}>0$. Since $\sigma_{I}<$ $s \rho /(1+\beta)$ and $\rho=(1-\beta \Delta)(1-s) / s$, we get $\sigma_{I}<1-s$. Consequently, in a model with inelastic labor supply, the existence of cycles and instability requires the capital income $(r k)$ to decrease when capital increases. A weak elasticity of intertemporal substitution in consumption $-1 / \varepsilon_{011}>0$ prevents the intertemporal arbitrage (see Becker and Foias $(1987,1994)$ ).

We further notice that, in contrast to the case with endogenous labor supply, the preferences of impatient consumers play no role on the stability properties of the steady state and the occurrence of cycles.

\subsection{Elastic labor supply}

The opposite case with elastic labor supply is now considered. Without a significant loss of generality, we assume that all the impatient agents $(i=1, \ldots, n)$ share the same instantaneous utility function and, in consequence, the same labor supply $l_{i} \equiv m$ as well as the same elasticities $\varepsilon_{i 11} \equiv \varepsilon_{11}, \varepsilon_{i 22} \equiv \varepsilon_{22}$. According to (72) (see the Appendix), their individual (and average) elasticity of labor supply becomes:

$$
\tilde{\xi}=-\frac{1+\varepsilon_{11}}{\varepsilon_{11}+\varepsilon_{22} m /(1-m)}
$$

while the capitalist's elasticity of labor supply with respect to the real wage is defined as above by $\xi_{0}=-\left(1-l_{0}\right) /\left(l_{0} \varepsilon_{022}\right)>0$. In the following, we consider two polar cases.

Assume first that the impatient agents' consumption is sufficiently substitutable over time $\left(-1 / \varepsilon_{11}>1\right)$. Hence, labor supply turns out to be positivesloped for all the agents. We notice that the more elastic the labor supplies (higher $\xi_{0}$ and $\left.\tilde{\xi}\right),{ }^{14}$ the weaker the bifurcation elasticity of capital-labor substitution $\sigma_{F}$ (see (43)). ${ }^{15}$ This means that the range of parameter compatible with the saddle-path stability $\left(\sigma_{F},+\infty\right)$ widens with the elasticity of labor supply and restrictions for instability and cycles become more demanding than under an exogenous labor supply.

If the impatient agents' intertemporal substitution in consumption is sufficiently weak $\left(-1 / \varepsilon_{11}<1\right)$, then, according to $(45), \tilde{\xi}$ becomes negative (while

\footnotetext{
${ }^{14}$ Notice that the less concave the disutilities of labor, the more elastic the labor supplies.

${ }^{15}$ In any case, $\sigma_{F}<\sigma_{I}$ because the term into brackets on the RHS of (43) is negative.
} 
$\xi_{0}$ still remains positive) and the critical elasticity $\sigma_{F}$ can be greater than $\sigma_{I}$. By direct inspection of equation (43), this requires:

$$
\tilde{\xi}<-\frac{\lambda_{0} \xi_{0}}{1-\lambda_{0}}\left(1+\frac{\rho}{2} \frac{3+\beta+\rho}{1+\beta}\right)
$$

We deduce that $\sigma_{F}>\sigma_{I}$ if the patient agent's labor supply is not too elastic and the negative aggregate elasticity $\tilde{\xi}$ not too close to zero, that is, the impatient agents' elasticity of intertemporal substitution in consumption $\left(-1 / \varepsilon_{11}\right)$ remains sufficiently weak. In this case, the introduction of a consumption-leisure arbitrage promotes instability and cycles, because the range of parameters for saddle-path stability $\left(\sigma_{F},+\infty\right)$ shrinks and the flip bifurcation occurs under a higher substitutability between capital and labor.

Now, let us provide two examples based on non-separable utility functions. Two popular specifications in macrodynamic literature are retained.

\section{KPR preferences}

In this section, we consider preferences à la King, Plosser and Rebelo (1988). They have the advantage of lightening restrictions on the structural parameters in order to obtain positive growth rates in models with capital accumulation. Agent $i$ 's utility function is specified as follows:

$$
\begin{aligned}
& u_{i}\left(c_{i}, 1-l_{i}\right) \equiv\left[c_{i} v_{i}\left(1-l_{i}\right)\right]^{1+\varepsilon_{i}} /\left(1+\varepsilon_{i}\right) \quad \text { if } \varepsilon_{i} \leq 0, \varepsilon_{i} \neq-1 \\
& u_{i}\left(c_{i}, 1-l_{i}\right) \equiv \ln c_{i}+\ln v_{i}\left(1-l_{i}\right) \text { if } \varepsilon_{i}=-1
\end{aligned}
$$

The parameter $-1 / \varepsilon_{i}$ is still interpretable as elasticity of intertemporal substitution in consumption. In addition, we need to define (first and second order) elasticities of leisure utility: $\varepsilon_{v_{i}} \equiv v_{i}^{\prime}\left(1-l_{i}\right)\left(1-l_{i}\right) / v_{i}\left(1-l_{i}\right)$ and $\varepsilon_{v_{i}^{\prime}} \equiv$ $v_{i}^{\prime \prime}\left(1-l_{i}\right)\left(1-l_{i}\right) / v_{i}^{\prime}\left(1-l_{i}\right)$. The KPR functional form (46) implies:

$$
\left[\begin{array}{ll}
\varepsilon_{i 11} & \varepsilon_{i 12} \\
\varepsilon_{i 21} & \varepsilon_{i 22}
\end{array}\right]=\left[\begin{array}{cc}
\varepsilon_{i} & \left(1+\varepsilon_{i}\right) \varepsilon_{v_{i}} \\
1+\varepsilon_{i} & \varepsilon_{v_{i}^{\prime}}+\varepsilon_{i} \varepsilon_{v_{i}}
\end{array}\right]
$$

Usual assumptions on preferences are maintained.

Assumption 4 (KPR) $v_{i}\left(1-l_{i}\right)$ is a continuous function on $[0,1]$, differentiable as many times as needed, increasing and concave on $(0,1)$, that is, $\varepsilon_{v_{i}^{\prime}} \leq 0 \leq \varepsilon_{v_{i}}$. We further assume:

$$
\left(1+2 \varepsilon_{i}\right) \varepsilon_{v_{i}}-\varepsilon_{i} \varepsilon_{v_{i}^{\prime}} \leq 0
$$

Assumption 4 deserves some comments. First, $\varepsilon_{v_{i}^{\prime}} \leq 0 \leq \varepsilon_{v_{i}}$ and $\varepsilon_{i} \leq 0$ imply $\varepsilon_{i 11} \leq 0, \varepsilon_{i 22} \leq 0$, while (49) is equivalent to $\varepsilon_{i 11} \varepsilon_{i 22} \geq \varepsilon_{i 12} \varepsilon_{i 21}$. So, concavity conditions (16) are met. Second, normality conditions (17) ensue from $\varepsilon_{v_{i}^{\prime}} \leq \varepsilon_{v_{i}}$.

Let us now define $Q \equiv\left(1-l_{0}\right)(1-\beta+\rho)+l\left(1-\beta+\rho \lambda_{0}\right)\left(\varepsilon_{v_{0}}-\varepsilon_{v_{0}^{\prime}}\right)$. Applying Proposition 4 to KPR preferences, we make the bifurcation through which cycles arise more explicit. 
Corollary 6 The system undergoes a flip bifurcation at $\sigma=\sigma_{F}>0$, if and only if both the inequalities are satisfied:

$$
\begin{aligned}
2 \varepsilon_{v_{0}}-\varepsilon_{v_{0}^{\prime}} & >\left(1-l_{0}\right)(1+\beta) /\left[\rho\left(l-l_{0}\right)\right] \\
-\frac{1}{\varepsilon_{0}} & <\frac{\left(l-l_{0}\right)\left(2 \varepsilon_{v_{0}}-\varepsilon_{v_{0}^{\prime}}\right)-\left(1-l_{0}\right)(1+\beta) / \rho}{\left(l-l_{0}\right) \varepsilon_{v_{0}}+\left(1-l_{0}\right)(1+\beta)+Q / 2}
\end{aligned}
$$

Otherwise saddle-path stability prevails whatever $\sigma>0$.

Proof. See the Appendix.

We observe that, when preferences are KPR, the elasticities of labor supply of impatient consumers $(i \geq 1)$ become zero, which implies in turn $\tilde{\xi}=0$. Therefore, preferences of impatient agents play no role on local dynamics, as it was the case under inelastic labor supply.

As seen above, a sufficiently low intertemporal substitution in patient agent's consumption $\left(-1 / \varepsilon_{0}\right)$ is needed to recover instability and the occurrence of endogenous cycles. This result is also in accordance with what happens under exogenous labor supply. However, by direct inspection of (51), we notice that a higher degree of concavity of $v_{0}$ (patient agent's utility of leisure) also promotes macroeconomic instability. Indeed, taken $\varepsilon_{v_{0}}$ as given, the left-hand side of (50) and the right-hand side of (51) increase with the degree of concavity $-\varepsilon_{v_{0}^{\prime}}$, making both the inequalities and instability more likely. Conversely, since the elasticity of labor supply with respect to the real wage $\xi_{0}=\left(1-l_{0}\right) /\left[l_{0}\left(\varepsilon_{v_{0}}-\varepsilon_{v_{0}^{\prime}}\right)\right]$ decreases with $-\varepsilon_{v_{0}^{\prime}}$, a more elastic labor supply of the patient agent promotes saddle-path stability and dynamic convergence.

\section{Homogeneous utilities}

One of the most popular classes of non-separable preferences is represented by homogeneous utility functions. In contrast to most existing papers, we don't reduce the analysis to homogeneity of degree one, but we allow the utility functions to have a degree less or equal to one.

The degrees of homogeneity and concavity are closely related. ${ }^{16}$ The former plays a key role on the stability properties, as the latter did in the KPR case. That's why taking into account a degree less than one matters.

Assumption 5 (homogeneity) The utility function $u_{i}\left(c_{i t}, 1-l_{i t}\right)$ is homogeneous of degree $\nu_{i} \leq 1$, for every $i=0, \ldots, n$.

Restriction $\nu_{i} \leq 1$ is needed to ensure the concavity and meet the second order conditions for intertemporal maximization. We also notice that CobbDouglas and CES utitlity functions satisfy Assumption 5, being homogeneous of degree one.

\footnotetext{
${ }^{16}$ The higher the degree of homogeneity, the lower the degree of concavity.
} 
As in the KPR case, the patient agent matters more than the others in the stability analysis. We will show, precisely, that the steady state becomes a saddle point as soon as the patient agent's degree $\nu_{0}$ becomes close to one, while, in contrast, the occurrence of cycles requires low degrees of homogeneity. In this connection, we need an additional notation.

$$
s_{i} \equiv u_{i 1} c_{i} / u_{i} \in(0,1)
$$

will denote the agent $i$ 's consumption share in total utility and $\varepsilon_{i} \equiv c_{i} u_{i 11} / u_{i 1}<$ 0 the elasticity of marginal utility with respect to consumption, while $-1 / \varepsilon_{i}$ will represent, as usual, the elasticity of intertemporal substitution in consumption.

Under Assumption 5, the homogeneity property and normality between consumption and leisure entail, respectively: ${ }^{17}$

$$
s_{i}<\nu_{i} \leq 1 \text { and } \varepsilon_{i} \nu_{i}+\left(1-\nu_{i}\right) s_{i}<0
$$

for every $i=0, \ldots, n$. Under properties (53), Proposition 4 is explicitly revisited.

Proposition 7 Proposition 4 still holds, where now:

$$
\begin{aligned}
\tilde{\xi} & =-\sum_{i=1}^{n} \frac{l_{i}\left(1-l_{i}\right)}{l-l_{0}}\left(1+\frac{\nu_{i}-s_{i}}{\varepsilon_{i} \nu_{i}+\left(1-\nu_{i}\right) s_{i}}\right) \\
\tilde{\xi}^{*} & =-\frac{1-l_{0}}{\left(1-\nu_{0}\right)\left(l-l_{0}\right)}\left[\rho+\varepsilon_{0} \frac{\nu_{0}-s_{0}}{\varepsilon_{0} \nu_{0}+\left(1-\nu_{0}\right) s_{0}}\right]<0 \\
\tilde{\xi}^{*}+\varkappa & =\tilde{\xi}^{*}+\frac{\rho}{1+\beta}\left[1-\frac{\rho+(1-\beta)\left(1+l-l_{0}\right)}{2\left(1-\nu_{0}\right)\left(l-l_{0}\right)}\right]
\end{aligned}
$$

Proof. See the Appendix.

Proposition 7 gives necessary and sufficient conditions for saddle-path stability, instability and deterministic cycles. In order to appreciate the economic intuition behind, we provide sufficient conditions for saddle-path stability in terms of the most significant parameter, the degree of homogeneity.

Corollary 8 Under Assumption 5, if the degree of homogeneity of the patient agent is sufficiently high, that is,

$$
\nu_{0}>1-\frac{\rho+(1-\beta)\left(1+l-l_{0}\right)}{2\left(l-l_{0}\right)}
$$

while the impatient agents' elasticities of intertemporal substitution in consumption are sufficiently large, that is,

$$
-1 / \varepsilon_{i}>1 /\left(1-s_{i}\right)
$$

for every $i=1, \ldots, n$, then the steady state is a saddle point (there is no room for cycles).

\footnotetext{
${ }^{17}$ See the proof of Proposition 7 in the Appendix
} 
Proof. See the Appendix.

Condition (58) is somewhat close to those we got above (see for instance, the separable case with elastic labor supply). Condition (57), which involves only the patient consumer's degree of homogeneity, is more puzzling and deserves great regard.

The next corollary stresses the prominence of (57), by asserting that restrictions (58) become superfluous to get saddle-path stability, when $\nu_{0}$ lies in a sufficiently small neighborhood of one.

Corollary 9 Under Assumption 5, when the capitalist's degree of homogeneity $\nu_{0}$ is sufficiently close to one the steady state is saddle-point stable and cycles are definitely ruled out.

In particular, when the preferences of the patient agent are homogeneous of degree one, saddle-path stability prevails.

Proof. See the Appendix.

Corollaries (8)-(9) undoubtedly show how the degree of homogeneity in the patient agent's utility matters to determine the stability properties of the economy. Homogeneous utilities of degree one, so popular in economic literature, have a very specific impact on dynamics and must be questioned on the ground of robustness. In this model, they imply dynamic convergence whatever the other parameter values, whereas stability is no longer ensured as soon as the degree of homogeneity lowers.

Eventually, we observe that lower the degree of homogeneity, higher the degree of concavity, more likely instability and cycles. We recover some results already found in the separable case (namely, under elastic labor supply) and in the KPR case. In both the cases the impact of concavity through the elasticity of labor supply emerges as dominant feature.

\section{Interpretation}

An economic intepretation of a bifurcation is a somewhat difficult task. In order to simplify this task, while preserving the main mechanism behind the emergence of cycles and the role of labor supply, it is not unworthy to compare the cases with elastic and inelastic labor supply.

The patient agent's budget constraint is the valuable information we need to start the analysis (see also Becker and Foias (1994)). In equilibrium, the constraints reduces to

$$
c_{0 t}+k_{t+1}-\Delta k_{t}=r_{t} k_{t}+w_{t} l_{0 t} \equiv I_{t}
$$

The economic intuition is based on the (possibly) negative response of the income $I_{t}$ to an increase in $k_{t}$. 
As announced, we first assume an inelastic labor supply: for simplicity, each agent supplies one unit of time. Therefore, $l_{0 t}=1$ and $l_{t}=1+n$. Using (18) and (19), we obtain:

$$
I_{t}=f\left(\frac{k_{t}}{1+n}\right)+f^{\prime}\left(\frac{k_{t}}{1+n}\right) \frac{n k_{t}}{1+n}
$$

We notice immediately that, in the case of a representative agent $(n=0), I_{t}$ is increasing in $k_{t}$. On the contrary, when consumers are heterogeneous $(n>0)$, $I_{t}$ can decrease in $k_{t}$, if $f^{\prime}\left(k_{t} /(1+n)\right) k_{t}$ lowers enough, which in turn requires a sufficiently weak capital-labor substitution, i.e., $\sigma<1-s$. In this case, the future capital stock $k_{t+1}$ decreases if the consumption $c_{0 t}$ is not too sensitive to changes in income $I_{t}$. This explains why a sufficiently weak elasticity of intertemporal substitution in consumption is necessary for the occurrence of cycles and instability and why concavity promotes cycles too.

Keeping in mind the basic mechanism, we can tackle the case of endogenous labor supply which makes more complex the income response to a change in capital.

The crucial question of the paper, we cannot get round, is why, in many cases, endogenous labor promotes saddle-path stability? Under elastic labor supply, the income $I_{t}$ becomes:

$$
I_{t}=f\left(a_{t}\right) l_{0 t}+f^{\prime}\left(a_{t}\right) a_{t} \sum_{i=1}^{n} l_{i t}
$$

with $l_{t}=\sum_{i=0}^{n} l_{i t}$ and $a_{t}=k_{t} / l_{t}$. All the labor supplies $l_{i t}(i=0,1, \ldots, n)$ depend on the real wage which is increasing in $k_{t}$. Therefore, an increase in $k_{t}$ generates two new effects: on the one hand, an impact on $I_{t}$ through $a_{t}$, that is, through $f\left(a_{t}\right)$ and $f^{\prime}\left(a_{t}\right) a_{t}$; on the other hand, an impact through the weights $l_{0 t}$ and $\sum_{i=1}^{n} l_{i t}$.

In order to evaluate the first effect, we observe that $l_{t}$ is an implicit function of $k_{t}$ and $c_{0 t}$ (equation (26)), and the capital-labor ratio is as well: $a_{t}=$ $k_{t} / l\left(k_{t}, c_{0 t}\right) \equiv a\left(k_{t}, c_{0 t}\right)$. Then, using (73) (see the Appendix), we obtain:

$$
\frac{\partial a / a}{\partial k / k}=\frac{\sigma(a) / s(a)}{\sigma(a) / s(a)+\lambda_{0} \xi_{0}+\left(1-\lambda_{0}\right) \widetilde{\xi}}
$$

As soon as $\lambda_{0} \xi_{0}+\left(1-\lambda_{0}\right) \widetilde{\xi}>0$, this elasticity is less than one, ${ }^{18}$ which means that, following an initial increase in $k_{t}$, the endogenous labor response reduces the increase of the capital-labor ratio and possibly dampens oscillations along non-monotonic capital paths.

In order to evaluate the second effect (on the weights $l_{0 t}$ and $\sum_{i=1}^{n} l_{i t}$ ), we consider the $a_{t}$ 's appearing directly in equation (60) as fixed. Under this restriction, the derivative of (60) with respect to $k_{t}$ is given by:

$$
k\left[f(a) \lambda_{0} \xi_{0}+a f^{\prime}(a)\left(1-\lambda_{0}\right) \tilde{\xi}\right] \frac{s(a)}{\sigma(a)} \frac{\partial a}{\partial k}
$$

\footnotetext{
${ }^{18}$ We notice that, when labor supply is inelastic, the capital-labor ratio is equal to $k /(1+n)$.
} 
The second effect also promotes stability if the income $I_{t}$ increases with $k_{t}$ through the weights $l_{0 t}$ and $\sum_{i=1}^{n} l_{i t}$. So, oscillations and endogenous cycles are dampened if (61) is positive, which depends on the sign of the term into the brackets and the sign of $\partial a / \partial k$. Both of them are positive if $\widetilde{\xi}$ is not too negative:

$$
\widetilde{\xi}>-\min \left\{\frac{\lambda_{0} \xi_{0}}{s(a)\left(1-\lambda_{0}\right)}, \frac{\lambda_{0} \xi_{0}+\sigma(a) / s(a)}{1-\lambda_{0}}\right\}
$$

The addition of both the effects can explain why, when the slope of the impatient agents' labor supply is not too negative or the degree of concavity of leisure utility is sufficiently weak, endogenous labor supply promotes saddle-path stability. One immediately sees that the same conclusion holds for the patient agent, through $\xi_{0}$.

Finally, let us notice that, as was the case under inelastic labor supply, endogenous cycles and instability occur more likely if $c_{0 t}$ is little sensitive to variations in $k_{t} \cdot{ }^{19}$ This requires a sufficiently weak elasticity of intertemporal substitution in consumption, a high degree of concavity of consumption utility or a degree of homogeneity sufficiently less than one.

\section{Conclusion}

We have addressed the question of saddle-path stability and dynamic convergence in an economy with heterogeneous consumers and elastic labor supply. In line with Becker (1980), Becker and Foias (1987, 1994), Hernandez (1991) and Sorger (1994), we have also supposed that agents face borrowing constraints, representing a kind of market incompleteness.

As in the case of inelastic labor supply, a state with a positive amount of capital in the hands of some impatient household cannot be stationary. This means that in a neighborhood of a steady state, the capital stock is wholly owned by the most patient agent, whereas the others consume their labor income. Focusing on such equilibria, we first establish the existence and uniqueness of the steady state.

At a second stage, we analyze the local dynamics to address the saddle-path stability and convergence issues. In particular, we focus on the role of preferences on the stability properties of the steady state and the occurrence of endogenous cycles. As was the case in economies with inelastic labor supply, instability and endogenous cycles require sufficiently weak capital-labor substitution. However, now, the taste for leisure and the impatient agents' behavior have a great influence on dynamics. We first derive conditions for saddle-path stability and the occurrence of flip bifurcation under general preferences in consumption and leisure. Then, we apply our results to particular classes of preferences widely employed in macrodynamics.

In order to fix ideas, it is not unworthy to recover the representative agent as a limit case. We find that the steady state is always a saddle point, in contrast

\footnotetext{
${ }^{19}$ We observe that, under elastic labor supply, $c_{0 t}$ also affects $l_{0 t}$ and, consequently, $l_{t}$ and $a_{t}$.
} 
to De Hek (1998) who obtains persistent fluctuations. However, his analysis is somewhat misleading, because founded on questionable assumptions such as the lack of normality in consumption and leisure.

Coming back to the model with heterogeneous consumers, we make explicit the role of preferences on dynamics, by presenting three classes of utility functions commonly used in economic literature.

When preferences are separable in consumption and leisure, the introduction of elastic labor supply promotes saddle-path stability if the impatient agents easily smooth consumption over time. If conversely, their intertemporal substitution in consumption is too weak, there is room for instability and deterministic cycles.

When preferences are KPR (King, Plosser and Rebelo (1988)), the impatient agents' utility functions cease from having any influence on dynamics, while only the patient agent's tastes matter. An elasticity of intertemporal substitution in consumption which is not too weak and a leisure utility which is not too concave are needed to ensure convergence, whatever the technological parameters.

Eventually, when preferences are homogeneous, the degree of homogeneity of the patient agent's utility naturally emerges as crucial parameter. In particular, we prove a somewhat intriguing result: when the degree is close to one, saddlepath stability prevails.

\section{Appendix}

Proof of Proposition 1. The $i$ th agent maximizes the intertemporal utility function (2) under the sequences of budget constraints (3) and borrowing constraints (4). The first order conditions reduce to the Euler equations:

$$
\frac{u_{i 1}\left(c_{i t}, 1-l_{i t}\right)}{u_{i 1}\left(c_{i t+1}, 1-l_{i t+1}\right)} \geq \beta_{i} R_{t+1}, \text { with equality if } k_{i t+1}>0
$$

the consumption-labor arbitrages: $u_{i 2}\left(c_{i t}, 1-l_{i t}\right)=u_{i 1}\left(c_{i t}, 1-l_{i t}\right) w_{t}$, the budget constraints (3) (now binding), and, eventually, the individual transversality conditions: $\lim _{t \rightarrow+\infty} \beta_{i}^{t} u_{i 1}\left(c_{i t}, 1-l_{i t}\right) k_{i t+1}=0$.

As seen above, heterogeneity in time preference leads the more patient agent to hold the entire capital stock near a stationary solution. More precisely, equation (62) becomes $R \leq 1 / \beta_{i}$ in a neighborhood of the steady state. But, since $\beta_{0}>\beta_{i}$ for $i>0$, the equilibrium interest factor will prevail, will be the cheapest rental factor:

$$
R=\min \left\{1 / \beta_{i}\right\}_{i=0}^{n}=1 / \beta_{0}
$$

(assumption (1)), and $1 / \beta_{i}>R$ at the stationary equilibrium for $i \geq 1$. Around the steady state, $u_{i 1}\left(c_{i t}, 1-l_{i t}\right) / u_{i 1}\left(c_{i t+1}, 1-l_{i t+1}\right)>\beta_{i} R_{t+1}$, for $i \geq 1$, according to equations (62) and (63). Firms borrow the cheapest capital from the individual $i=0$, while individuals $i \geq 1$ end up to hold no capital: $k_{0 t}>0$ and $k_{i t}=0$ for $i \geq 1$. 
The capitalist's equation $(62)(i=0)$ will hold with equality, jointly with the consumption-leisure arbitrage (9), the budget constraint (10) and the transversality condition.

The impatient agents' budget constraints (3) simplify to (11), because now $k_{i t}=0$ (for $i \geq 1$ ). The consumption-leisure arbitrages (9) still hold, where $c_{i t}$ is given by (11). Eventually, the transversality conditions are satisfied.

Inequalities (13) must hold at every period in order to ensure the capitalistic distribution as an intertemporal equilibrium.

Proof of Lemma 2. First, we prove that $l_{0}\left(w, c_{0}\right)$ is well defined from:

$$
\varphi_{0}\left(l_{0}, c_{0}\right)=w
$$

where $\varphi_{0}\left(l_{0}, c_{0}\right) \equiv u_{02}\left(c_{0}, 1-l_{0}\right) / u_{01}\left(c_{0}, 1-l_{0}\right)$. We observe that under Assumption $1, \lim _{l_{0} \rightarrow 1^{-}} \varphi_{0}\left(l_{0}, c_{0}\right)=+\infty$. $\varphi_{0}$ crosses at least once $w$ because, by hypothesis, $\lim _{l_{0} \rightarrow 0} u_{02}\left(c_{0}, 1-l_{0}\right) / u_{01}\left(c_{0}, 1-l_{0}\right)<w$. Moreover, under Assumption 2, $\partial \varphi_{0}\left(l_{0}, c_{0}\right) / \partial l_{0}=-\left(u_{022}-u_{012} u_{02} / u_{01}\right) / u_{01}>0$. Then, $\varphi_{0}$ crosses $w$ only once and the solution of (64) is unique.

Second, we prove the existence of the function $l_{i}(w)$ for $i \geq 1$. We need to solve the implicit equation:

$$
\varphi_{i}\left(l_{i}\right)=w
$$

where, as above, $\varphi_{i}\left(l_{i}\right) \equiv u_{i 2}\left(w l_{i}, 1-l_{i}\right) / u_{i 1}\left(w l_{i}, 1-l_{i}\right)$. Given $w$, we have, under Assumption 1, $\lim _{l_{i} \rightarrow 0^{+}} \varphi_{i}\left(l_{i}\right)=0$ and $\lim _{l_{i} \rightarrow 1^{-}} \varphi_{i}\left(l_{i}\right)=+\infty$. By continuity, at least a solution of equation (65) exists. In addition, since $\varphi_{i}^{\prime}\left(l_{i}\right)=$ $\left[u_{i 12} u_{i 2} / u_{i 1}-u_{i 22}+w\left(u_{i 21}-u_{i 11} u_{i 2} / u_{i 1}\right)\right] / u_{i 1}>0$ under Assumption 2, the function $\varphi_{i}$ crosses $w$ only once. The solution $l_{i}(w)$ of equation (65) is thus unique.

Proof of Proposition 3. As a preliminary result, we observe that, under Assumption 1, the labor supply of each consumer is always smaller than one, i.e., $l_{i}<1$ for all $i \geq 0$. Now, notice that a steady state is defined by:

$$
\begin{aligned}
r(k / l) & =1 / \beta-\Delta \\
l & =l_{0}\left(w(k / l), c_{0}\right)+\sum_{i=1}^{n} l_{i}(w(k / l)) \\
c_{0} & =(r(k / l)-\delta) k+w(k / l) l_{0}\left(w(k / l), c_{0}\right)
\end{aligned}
$$

Under Assumption 3, there exists a unique solution $k / l \equiv \alpha$ to the first equation, where $\alpha>0$ is a constant. The wage $w(k / l)=w(\alpha)$ is constant too and the functions $l_{i}(w)$ as well (denote them $\bar{l}_{i}$ ). Finally, the agent 0 's labor supply is defined by:

$$
u_{02}\left((1 / \beta-1) k+w l_{0}, 1-l_{0}\right)=w u_{01}\left((1 / \beta-1) k+w l_{0}, 1-l_{0}\right)
$$


and determines a continuous function $l_{0}=\hat{l}_{0}(k)$, with $k=\alpha l$. Since the sum of continuous functions is continuous,

$$
\lambda(l) \equiv \hat{l}_{0}(\alpha l)+\sum_{i=1}^{n} \bar{l}_{i}
$$

is continuous. A steady state is a solution to the equation $l=\lambda(l)$, where $l$ belongs to $(0,1+n)$. Using our preliminary result, we deduce that $\lambda(0)<1+n$. Moreover, we have:

$$
\lambda^{\prime}(l)=-\alpha \frac{1-\beta}{\beta}\left(w+\frac{c_{0}}{1-l_{0}} \frac{\varepsilon_{022}-\varepsilon_{012}}{\varepsilon_{011}-\varepsilon_{021}}\right)^{-1}<0,
$$

according to (17). Thus, there is a unique solution $l^{*}=\lambda\left(l^{*}\right)$, which is a steady state and determines the other variables, namely $k^{*}$ and $l_{0}^{*}$.

Local Dynamics. In order to get local information on dynamics, we linearize the dynamic system (27)-(28) around the steady state. Equations (27) and (28) become, respectively:

$$
\begin{aligned}
& -\frac{u_{012}\left(1-l_{0}\right)}{u_{01}} \frac{l_{0}}{1-l_{0}} \frac{l_{0 w} w}{l_{0}}\left(\frac{w_{k} k}{w}+\frac{w_{l} l}{w} \frac{l_{k} k}{l}\right) \frac{d k_{t}}{k} \\
& +\left[\frac{u_{011} c_{0}}{u_{01}}-\frac{u_{012}\left(1-l_{0}\right)}{u_{01}} \frac{l_{0}}{1-l_{0}}\left(\frac{l_{0 c_{0}} c_{0}}{l_{0}}+\frac{l_{0 w} w}{l_{0}} \frac{w_{l} l}{w} \frac{l_{c_{0}} c_{0}}{l}\right)\right] \frac{d c_{0 t}}{c_{0}} \\
& +\left[\frac{u_{012}\left(1-l_{0}\right)}{u_{01}} \frac{l_{0}}{1-l_{0}} \frac{l_{0 w} w}{l_{0}}\left(\frac{w_{k} k}{w}+\frac{w_{l} l}{w} \frac{l_{k} k}{l}\right)-\beta r\left(\frac{r_{k} k}{r}+\frac{r_{l} l}{r} \frac{l_{k} k}{l}\right)\right] \frac{d k_{t+1}}{k} \\
& -\left[\frac{u_{011} c_{0}}{u_{01}}-\frac{u_{012}\left(1-l_{0}\right)}{u_{01}} \frac{l_{0}}{1-l_{0}}\left(\frac{l_{0 c_{0}} c_{0}}{l_{0}}+\frac{l_{0 w} w}{l_{0}} \frac{w_{l} l}{w} \frac{l_{c_{0}} c_{0}}{l}\right)+\beta r \frac{r_{l} l}{r} \frac{l_{c_{0}} c_{0}}{l}\right] \frac{d c_{0 t+1}}{c_{0}} \\
& =0
\end{aligned}
$$

and

$$
\begin{aligned}
& \left(\left[\Delta+\left(1+\frac{r_{k} k}{r}+\frac{r_{l} l}{r} \frac{l_{k} k}{l}\right) r\right] k+w l_{0}\left(\frac{w_{k} k}{w}+\frac{w_{l} l}{w} \frac{l_{k} k}{l}\right)\left(1+\frac{l_{0 w} w}{l_{0}}\right)\right) \frac{d k_{t}}{k} \\
& +\left(\frac{r_{l} l}{r} \frac{l_{c_{0}} c_{0}}{l} r k+w l_{0}\left[\frac{l_{0 c_{0}} c_{0}}{l_{0}}+\frac{w_{l} l}{w} \frac{l_{c_{0}} c_{0}}{l}\left(1+\frac{l_{0 w} w}{l_{0}}\right)\right]-c_{0}\right) \frac{d c_{0 t}}{c_{0}} \\
& -k \frac{d k_{t+1}}{k}=0
\end{aligned}
$$

To achieve the reduction in elasticities, we need those of labor supply. We know that $l_{0}=l_{0}\left(w, c_{0}\right), l_{i}^{*}=l_{i}(w)$ and $l=l\left(k, c_{0}\right)$ are respectively given by (9), (23) and (25). Total differentiating (9), we get:

$$
\begin{aligned}
\varepsilon_{w 0} & \equiv \frac{l_{0 w} w}{l_{0}}=\frac{1-l_{0}}{l_{0}} \frac{1}{\varepsilon_{012}-\varepsilon_{022}} \equiv \xi_{0}>0 \\
\varepsilon_{c 0} & \equiv \frac{l_{0 c_{0}} c_{0}}{l_{0}}=\frac{1-l_{0}}{l_{0}} \frac{\varepsilon_{021}-\varepsilon_{011}}{\varepsilon_{022}-\varepsilon_{012}}<0
\end{aligned}
$$


Totally differentiating (23), we obtain:

$$
\xi_{i} \equiv \frac{l_{i}^{* \prime}(w) w}{l_{i}}=\frac{1+\varepsilon_{i 11}-\varepsilon_{i 21}}{\varepsilon_{i 21}-\varepsilon_{i 11}+\left(\varepsilon_{i 12}-\varepsilon_{i 22}\right) l_{i} /\left(1-l_{i}\right)}
$$

for $i=1, \ldots, n$, while, totally differentiating (25), we find:

$$
\begin{aligned}
\frac{l_{k} k}{l} & =\frac{\lambda_{0} \varepsilon_{w 0}+\sum_{i=1}^{n} \lambda_{i} \xi_{i}}{\lambda_{0} \varepsilon_{w 0}+\sum_{i=1}^{n} \lambda_{i} \xi_{i}+\sigma / s} \\
\frac{l_{c_{0}} c_{0}}{l} & =\frac{\lambda_{0} \varepsilon_{c 0} \sigma / s}{\lambda_{0} \varepsilon_{w 0}+\sum_{i=1}^{n} \lambda_{i} \xi_{i}+\sigma / s}
\end{aligned}
$$

where $\lambda_{i}$ is the individual labor share in total labor supply. Substituting the elasticities (70), (71), (73) and (74) in equations (68) and (69), we get a reduced system:

$$
\begin{gathered}
-(1+\rho x) \frac{d k_{t+1}}{k}+\left[\lambda_{0} \varepsilon_{c 0}(1+\rho x)-z\left(\varepsilon_{011} \varepsilon_{022}-\varepsilon_{012} \varepsilon_{021}\right) / \varepsilon_{012}\right] \frac{d c_{0 t+1}}{c_{0}} \\
=-\frac{d k_{t}}{k}+\left[\lambda_{0} \varepsilon_{c 0}-z\left(\varepsilon_{011} \varepsilon_{022}-\varepsilon_{012} \varepsilon_{021}\right) / \varepsilon_{012}\right] \frac{d c_{0 t}}{c_{0}} \\
-\beta \frac{d k_{t+1}}{k}=\left[\rho\left(1-\lambda_{0}-\lambda_{0} \varepsilon_{w 0}\right) / z-1\right] \frac{d k_{t}}{k} \\
+\left[\rho \lambda_{0}\left(1-\varepsilon_{c 0}\left[1+\left(1-\lambda_{0}-\lambda_{0} \varepsilon_{w 0}\right) / z\right]\right)+1-\beta\right] \frac{d c_{0 t}}{c_{0}}
\end{gathered}
$$

where

$$
\begin{aligned}
x & \equiv 1-\varepsilon_{022} / \varepsilon_{012} \\
z & \equiv \sigma / s+\xi
\end{aligned}
$$

Afterwards, we evaluate the Jacobian matrix $J$ of system (27)-(28) at the steady state:

$$
\begin{aligned}
& {\left[\begin{array}{cc}
-(1+\rho x) & \lambda_{0} \varepsilon_{c 0}(1+\rho x)-z\left(\varepsilon_{011} \varepsilon_{022}-\varepsilon_{012} \varepsilon_{021}\right) / \varepsilon_{012} \\
-\beta & 0
\end{array}\right]^{-1}} \\
& {\left[\begin{array}{cc}
-1 & \lambda_{0} \varepsilon_{c 0}-z\left(\varepsilon_{011} \varepsilon_{022}-\varepsilon_{012} \varepsilon_{021}\right) / \varepsilon_{012} \\
\rho\left(1-\lambda_{0}-\lambda_{0} \varepsilon_{w 0}\right) / z-1 & \rho \lambda_{0}\left(1-\varepsilon_{c 0}\left[1+\left(1-\lambda_{0}-\lambda_{0} \varepsilon_{w 0}\right) / z\right]\right)+1-\beta
\end{array}\right]}
\end{aligned}
$$

We deduce the trace $T$ and the determinant $D$ of the Jacobian matrix using (75) and (76).

Proof of Corollary 6. We apply the results obtained in Proposition 4. Using (48), the critical values $\tilde{\xi}^{*}$ and $\varkappa$ are explicitly computed:

$$
\begin{aligned}
\tilde{\xi}^{*} & =\frac{1-l_{0}}{l-l_{0}} \frac{\rho-\varepsilon_{0}}{\left(1+2 \varepsilon_{0}\right) \varepsilon_{v_{0}}-\varepsilon_{0} \varepsilon_{v_{0}^{\prime}}}(<0) \\
\varkappa & =\frac{\rho}{1+\beta}\left(1+\frac{Q}{2\left(l-l_{0}\right)\left[\left(1+2 \varepsilon_{0}\right) \varepsilon_{v_{0}}-\varepsilon_{0} \varepsilon_{v_{0}^{\prime}}\right]}\right)
\end{aligned}
$$


Since $\xi_{i}=0$ for all $i \geq 1$ (expression (72)), we have also $\tilde{\xi}=0$. As highlighted in Proposition 4, a flip bifurcation generically arises if and only if $\sigma_{F}=s\left(1-\lambda_{0}\right)\left(\tilde{\xi}^{*}+\varkappa\right)>0$, which requires $\tilde{\xi}^{*}+\varkappa>0$. Using (49), (77) and (78), this last inequality holds if and only if (50) and (51) are satisfied.

Proof of Proposition 7. Since the utility function $u_{i}$ is homogeneous of degree $\nu_{i}, u_{i}\left(\mu c_{i}, \mu\left(1-l_{i}\right)\right)=\mu^{\nu_{i}} u_{i}\left(c_{i}, 1-l_{i}\right)$ for any positive real number $\mu$. Homogeneity implies some well-known properties: $s_{i 1}+s_{i 2}=\nu_{i}, \varepsilon_{i 11}+\varepsilon_{i 12}=$ $\nu_{i}-1=\varepsilon_{i 21}+\varepsilon_{i 22}$ and

$$
\frac{s_{i 1}}{s_{i 2}}=\frac{\nu_{i}-1-\varepsilon_{i 22}}{\nu_{i}-1-\varepsilon_{i 11}}
$$

for $i=0, \ldots, n$, where $s_{i 1} \equiv u_{i 1} c_{i} / u_{i}$ and $s_{i 2} \equiv u_{i 2}\left(1-l_{i}\right) / u_{i}$ are the consumption and leisure shares in total utility and $\varepsilon_{i j 1}$ and $\varepsilon_{i j 2}$ the elasticities of marginal utilities (definition (15)). ${ }^{20}$

In our analysis, the key variables are the consumption share in total utility $s_{i 1}$ and the elasticity of intertemporal substitution in consumption $\left(-1 / \varepsilon_{i 11}\right)$. Simplifying notation as follows: $\varepsilon_{i} \equiv \varepsilon_{i 11}$ and $s_{i} \equiv s_{i 1}$, we get $s_{i 2}=\nu_{i}-s_{i}$ and:

$$
\begin{aligned}
\varepsilon_{i 12} & =\nu_{i}-1-\varepsilon_{i} \\
\varepsilon_{i 21} & =\frac{s_{i}}{\nu_{i}-s_{i}}\left(\nu_{i}-1-\varepsilon_{i}\right) \\
\varepsilon_{i 22} & =\nu_{i}-1-\frac{s_{i}}{\nu_{i}-s_{i}}\left(\nu_{i}-1-\varepsilon_{i}\right)
\end{aligned}
$$

The first inequality in (53) ensues from $s_{i 1}, s_{i 2} \in\left(0, \nu_{i}\right)$. From $(80),(81)$ and (82), we compute other expressions of interest appearing in $\tilde{\xi}^{*}, \tilde{\xi}^{*}+\varkappa$ and $\tilde{\xi} \equiv \sum_{i=1}^{n} \lambda_{i} \xi_{i} /\left(1-\lambda_{0}\right)$, that is in equations (32), (33) and (72), respectively:

$$
\begin{aligned}
\varepsilon_{i 11}-\varepsilon_{i 21} & =\left[\varepsilon_{i} \nu_{i}+\left(1-\nu_{i}\right) s_{i}\right] /\left(\nu_{i}-s_{i}\right)=\varepsilon_{i 22}-\varepsilon_{i 12} \\
\varepsilon_{i 11} \varepsilon_{i 22}-\varepsilon_{i 12} \varepsilon_{i 21} & =-\left(1-\nu_{i}\right)\left(\varepsilon_{i 11}-\varepsilon_{i 21}\right)
\end{aligned}
$$

Normality conditions (16) and (17) immediately entail the second inequality in (53). Substituting (80)-(84) into (32), (33) and (72), we eventually obtain (54), (55) and (56).

Proof of Corollary 8. Saddle-path stability requires $\sigma>\sigma_{F}$. Then $\sigma_{F}<0$ or, equivalently $\tilde{\xi} \geq \tilde{\xi}^{*}+\varkappa$, implies convergence. Replacing (54), (55) and (56)

\footnotetext{
${ }^{20}$ These properties are derived using the Euler's law $\left(u_{i 1} c_{i}+u_{i 2}\left(1-l_{i}\right)=\nu u_{i}\right)$ and the fact that the marginal utilities are homogeneous of degree $\nu_{i}-1$. Equation (79) is obtained differentiating the Euler's law w.r.t. to $c_{i}$ and $1-l_{i}$ and noticing that the cross derivatives are equal.
} 
in $\tilde{\xi} \geq \tilde{\xi}^{*}+\varkappa$, we get

$$
\begin{aligned}
& \sum_{i=1}^{n} l_{i}\left(1-l_{i}\right)\left(1+\frac{\nu_{i}-s_{i}}{\varepsilon_{i} \nu_{i}+\left(1-\nu_{i}\right) s_{i}}\right) \\
\leq & \frac{1-l_{0}}{1-\nu_{0}}\left(\rho+\varepsilon_{0} \frac{\nu_{0}-s_{0}}{\varepsilon_{0} \nu_{0}+\left(1-\nu_{0}\right) s_{0}}\right) \\
& -\frac{\rho}{1+\beta}\left(l-l_{0}-\frac{\rho+(1-\beta)\left(1+l-l_{0}\right)}{2\left(1-\nu_{0}\right)}\right)
\end{aligned}
$$

A sufficient condition for the left-hand side in inequality (85) to be negative is $1+\left(\nu_{i}-s_{i}\right) /\left[\varepsilon_{i} \nu_{i}+\left(1-\nu_{i}\right) s_{i}\right]<0$ for every $i$, that is (58) under (53). A sufficient condition for the right-hand side to be positive is $l-l_{0}-$ $\left[\rho+(1-\beta)\left(1+l-l_{0}\right)\right] /\left[2\left(1-\nu_{0}\right)\right]<0$, that is $(57)$ under $(53)$.

Proof of Corollary 9. We notice that, under (53), as $\nu_{0}$ tends to $1^{-}$, the lefthand side of inequality (85) in the Proof of Corollary 8 gets a finite value, while the right-hand side goes to $+\infty$. Then inequality (85) asymptotically holds.

\section{References}

[1] Barro, R. and X. Sala-i-Martin (1995), Economic Growth, New-York, McGraw-Hill.

[2] Becker, R. A. (1980), On the Long-Run Steady State in a Simple Dynamic Model of Equilibrium with Heterogeneous Households, Quarterly Journal of Economics 95, 375-382.

[3] Becker, R. A. and C. Foias (1987), A Characterization of Ramsey Equilibrium, Journal of Economic Theory 41, 173-184.

[4] Becker, R. A. and C. Foias (1994), The Local Bifurcation of Ramsey Equilibrium, Economic Theory 4, 719-744.

[5] Bosi, S., F. Magris and A. Venditti (2005), Competitive Equilibrium Cycles with Endogenous Labor, Journal of Mathematical Economics 41, 325-349.

[6] De Hek, P. A. (1998), An Aggregative Model of Capital Accumulation with Leisure-dependent Utility, Journal of Economic Dynamics and Control 23, $255-276$.

[7] Garcia-Penalosa, C. and S. J. Turnovsky (2006), Growth and Income Inequality: A Canonical Model, Economic Theory 28, 28-49.

[8] Garnier, J.-P., K. Nishimura and A. Venditti (2006), Intertemporal Substitution in Consumption, Labor Supply Elasticity and Capital-labor Substitution in Continuous-time Models, miméo., Aix-Marseille. 
[9] Ghiglino, C. and G. Sorger (2002), Poverty Traps, Indeterminacy, and the Wealth Distribution, Journal of Economic Theory 105, 120-139.

[10] Grandmont, J.-M., P. Pintus and R. de Vilder (1998), Capital-labour Substitution and Competitive Nonlinear Endogenous Business Cycles, Journal of Economic Theory 80, 14-59.

[11] Hernandez, A. (1991), The Dynamics of Competitive Equilibrium Allocations with Borrowing Constraints, Journal of Economic Theory 55, 180191.

[12] King, R., C. Plosser and S. Rebelo (1988), Production, Growth and Business Cycles, Journal of Monetary Economics 21, 191-232.

[13] Ladron-de-Guevara, A., S. Ortiguiera and M. S. Santos (1997), Equilibrium Dynamics in Two-sector Models of Endogenous Growth, Journal of Economic Dynamics and Control 21, 115-143.

[14] Le Van, C., N. Manh Hung and Y. Vailakis (2006), Equilibrium Dynamics in an Aggregative Model of Capital Accumulation with Heterogeneous Agents and Elastic Labor, Journal of Mathematical Economics, forthcoming.

[15] Nourry, C. (2001), Stability of Equilibria in the Overlapping Generations Model with Endogenous Labor Supply, Journal of Economic Dynamics and Control 25, 1647-1663.

[16] Nourry, C. and A. Venditti (2006), Overlapping Generations Model with Endogenous Labor Supply: General Formulation, Journal of Optimization Theory and Applications 128, 355-377.

[17] Pintus, P. (2006), Indeterminacy with Almost Constant Returns to Scale: Capital-labor Substitution Matters, Economic Theory 28, 633-49.

[18] Sorger, G. (1994), On the Structure of Ramsey Equilibrium: Cycles, Indeterminacy, and Sunspots, Economic Theory 4, 745-764.

[19] Sorger, G. (2000), Income and Wealth Distribution in a Simple Model of Growth, Economic Theory 16, 23-42. 